\title{
Electromagnetic heavy-lepton pair production in relativistic heavy-ion collisions
}

\author{
M. Y. Şengüil ${ }^{1, a}$, M. C. Güçlï ${ }^{2}$ Ö. Mercan ${ }^{2}$, N. G. Karakuş ${ }^{2}$ \\ ${ }_{1}^{1}$ Atakent Mahallesi, 3. Etap, 34303 Halkali-Küçükçekmece, Istanbul, Turkey \\ ${ }^{2}$ Faculty of Science and Letters, İstanbul Technical University, 34469 Istanbul, Turkey
}

Received: 27 August 2015 / Accepted: 15 July 2016 / Published online: 1 August 2016

(C) The Author(s) 2016. This article is published with open access at Springerlink.com

\begin{abstract}
We calculate the cross sections of electromagnetic productions of muon- and tauon-pair productions from the ultra-relativistic heavy ion collisions. Since the Compton wavelengths of muon and tauon are comparable to the radius of the colliding ions, nuclear form factors play important roles for calculating the cross sections. Recent measurement (Abrahamyan et al., Phys Rev Lett 108:112502, 2012) indicates that the neutrons are differently distributed from the protons; therefore this affects the cross section of the heavylepton pair production. In order to see the effects of the neutron distributions in the nucleus, we used analytical expression of the Fourier transforms of the Wood-Saxon distribution. Cross section calculations show that the Wood-Saxon distribution function is more sensitive to the parameter $R$ compared to the parameter $a$.
\end{abstract}

\section{Introduction}

Colliding beams of heavy ions at energies per nucleon in the range $10^{2}-10^{4} \mathrm{GeV}$, corresponding to the RHIC experiments at Brookhaven, and LHC at CERN, suggested that intense electromagnetic fields of these ions can produce relatively large fluxes of muons and tauons. Since the mass of the electron $(0.511 \mathrm{MeV})$ is much smaller than the muon $(105.66 \mathrm{MeV})$ and the tauon $(1784 \mathrm{MeV})$ masses, and the Compton wavelength of the electron $(386 \mathrm{fm})$ is much larger than the muon $(1.86 \mathrm{fm})$ and the tauon $(0.11 \mathrm{fm})$ masses, the cross sections of producing electron pairs are much larger than the heavy leptons. In addition to this, since the Compton wavelengths of muons and tauons are smaller than the radius of the colliding heavy ions $(\mathrm{Au}, \mathrm{Pb})$, the nucleon [1] and nucleus form factors are not negligible. Therefore realistic charge form factors play important roles for calculating the cross sections of the heavy-lepton pair productions.

\footnotetext{
a e-mail: melekaurora@yahoo.com
}

In this work, we have used the Wood-Saxon nucleus form factor which is also widely used in the literature [2-5]. Instead of the monopole approximation, we have an analytical expression of the Wood-Saxon form factor in our equations. With the help of this analytical equation, we are able to account for the neutron skin and its effects to our calculations. The determination of the sizes and shapes of atomic nuclei is one of the important problems in nuclear physics. The rms radius of the charge distribution in nuclei is well known, with a high degree of accuracy achieved by the elastic electron-nucleus and muon-nucleus scattering experiments. The experimental uncertainties for the nuclear charge radius are about $1 \%$ for several nuclei [6]. However, the neutron distribution and its rms radius in nuclei is not precisely determined theoretically and experimentally. Generally, the neutron skin thickness is defined as the neutron-proton rms radius difference in the atomic nucleus,

$$
\Delta R_{n p}=R_{n}-R_{p}=\left\langle r^{2}\right\rangle_{n}^{1 / 2}-\left\langle r^{2}\right\rangle_{p}^{1 / 2}
$$

where the measured charge radius is $\left\langle r^{2}\right\rangle_{p}^{1 / 2}=5.43 \mathrm{fm}$ for the gold nucleus and $\left\langle r^{2}\right\rangle_{p}^{1 / 2}=5.50 \mathrm{fm}$ for the lead nucleus [2]. By using the parity-violating electron scattering (PVES), the recent experiment [7] at JLab gives us the value $R_{n}$ as $5.78_{-0.18}^{+0.16}$. The difference between the radii of the neutron and proton distributions is the neutron skin and it is equal to $R_{\text {skin }}=R_{n}-R_{p}=0.33_{-0.18}^{+0.16}$ fm for the lead nucleus with large total error. Therefore the difference between the distributions of protons and neutrons in the nucleus affects the cross section of the heavy-lepton pair production.

To explore a surface thickness of the neutron density in a heavy nucleus, we model the neutron density with a WoodSaxon form:

$\rho_{n}(r)=\rho_{0} /\left[1+\exp \left(r-R_{0}\right) / a_{n}\right]$

where $R_{0}$ and $a_{n}$ are the parameters that determine the shape of the neutron distribution. Although the same parameters are 


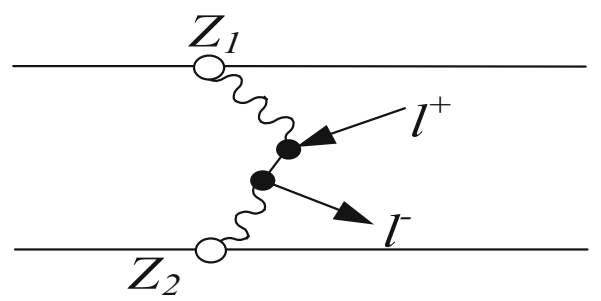

(a)

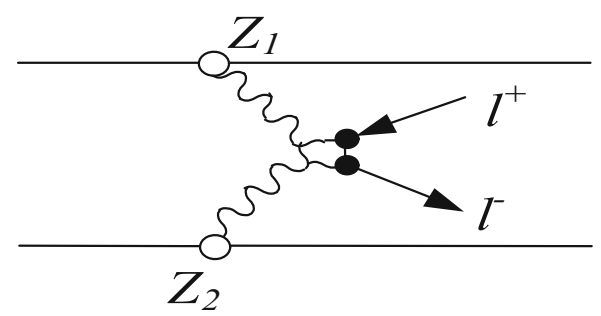

Fig. 1 a Direct and $\mathbf{b}$ crossed Feynman diagrams for the heavy-lepton pair production in a relativistic heavy ion collision

precisely determined for the proton distribution in the heavy nucleus, there are large uncertainties for the neutron distribution. In this work, we study the effect of these parameters on the heavy-lepton pair production cross sections.

Although the electron-positron pair production from electromagnetic fields goes back to the 1930s [8,9], there are values in the published literature in the 1990s and 2000s [10-16]. The main motivation at this period was the RHIC. Dilepton production from the central collisions of heavy ions is very important to understand the hadronic interactions. However, electromagnetic production of dileptons from the peripheral collisions of heavy ions can shield those dileptons produced from the hadronic interactions. Therefore it is highly important to understand various properties of the electromagnetically produced dileptons [17].

In our previous work $[11,18-20]$, we have calculated electron-positron pair production cross sections by using the second order Feynman diagrams. In this work, we have modified the equations by including the electromagnetic form factors of nuclei in the momentum space. We have used Fermi type charge form factor. We also consider the coherent production of heavy-lepton pairs in two-photon limit for the symmetric collision of two gold nuclei at RHIC and two lead nuclei at LHC energies. In Fig. 1, the wiggly lines are the virtual photons that make the electromagnetic field of the colliding ions. Here we start with the lowest non-vanishing Feynman diagram which is called a two-photon diagram. We take the classical limit of the motion of the ions, which yields the external field model. Since the parameter $Z \alpha \approx 0.6$ is not small, Coulomb and unitarity corrections should be included to obtain exact cross sections. This calculation has been done in [21-23], and we will also mention it briefly in the results section.

In Sect. 2, we briefly explain the two-photon pair production mechanism. In Sect. 3, we study the effects of the form

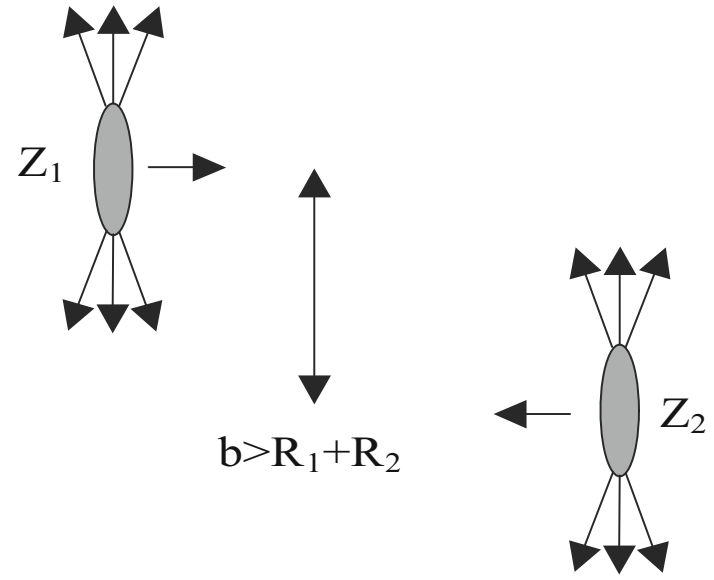

Fig. 2 Collision of two heavy ions at ultra-relativistic velocities. Lorentz-contracted strong EM fields are produced around the ions

factor that we use in our calculations and we also calculate a variety of cross sections for the single-pair process. Finally on Sect. 4, we summarize all the work that we have done in this manuscript.

\section{Calculations and results}

In this work we will investigate the two-photon mechanism for heavy-lepton pair production. In Fig. 2, the electromagnetic interaction of the colliding ions is shown. Since all the protons in the nucleus act coherently, there is a very strong electromagnetic field appearing around the heavy ions [10]. Lepton pairs are produced from this field, which is a cloud of virtual photons. Although we consider peripheral collisions of the heavy-ions where the impact parameter $b>R_{1}+R_{2}$ is greater than the sum of the radii of the colliding nuclei, our cross section expressions include the whole impact parameter range from zero to infinity. We consider the electromagnetic production of lepton pairs from the collisions of heavy ions with charge $Z$,

$Z_{1}+Z_{2} \rightarrow Z_{1}+Z_{2}+l^{+} l^{-}$,

where $l^{+} l^{-}$can be electrons, muons or tauons. The maximum magnitude of the electric field of the ions is

$E_{\max } \simeq \frac{Z e \gamma}{b^{2}}$

where $\gamma$ is the Lorentz factor, $b$ is the impact parameter, and $Z e$ is the charge of the fully stripped heavy ion. For the impact parameters around $b \sim 7.5 \mathrm{fm}$, which is very close to the nucleus but still concerns peripheral collisions, the maximum strength of the electric field at the surface is $E_{\max } \sim 2 \times 10^{23} \mathrm{~V} / \mathrm{m}$ for the RHIC and $E_{\max } \sim 7.14 \times$ $10^{24} \mathrm{~V} / \mathrm{m}$ for LHC collisions. We can estimate the minimum electric field that can create a lepton pair of mass $2 m c^{2}$ : 
$E_{\min } \simeq \frac{m^{2} c^{4}}{e \hbar c}$

where the distance that we used in the above equation is twice the Compton wavelength of the particular lepton [11]. To create $e^{+} e^{-}, \mu^{+} \mu^{-}$, and $\tau^{+} \tau^{-}$pairs, the minimum electric fields needed are $1.32 \times 10^{18}, 5.66 \times 10^{22}$ and $1.62 \times 10^{25} \mathrm{~V} / \mathrm{m}$, respectively. We see that, even at LHC energies, the maximum electric field produced from the lead ions is below the critical electric field to create tauon pairs. Even for the impact parameters around the $b \sim 7.5 \mathrm{fm}-30 \mathrm{fm}$ ranges, the maximum electric field is smaller than the electric field that produces tauon pairs at RHIC and LHC energies. However, in QED there is still a probability to produce heavy leptons even at these energies.

The source currents for producing lepton pairs are the Lorentz-boosted charges of the colliding heavy ions. Electromagnetic fields in these collisions are very strong; they can pull many lepton pairs from the vacuum. Relativistic heavy-ion collider (RHIC) at Brookhaven can accelerate the fully stripped gold nuclei at $100 \mathrm{GeV} /$ nucleon energies and the large hadron collider (corresponding to run-2 at LHC) can accelerate the protons up to energies of $E_{p}=6.5 \mathrm{TeV}$. The fully stripped lead nuclei can be accelerated at LHC run2 up to $E_{p} Z / A \approx 2.5 \mathrm{TeV} /$ nucleon energies, which yields $\sqrt{s} \approx 5.0 \mathrm{TeV} /$ nucleon. In the literature, most of the calculations about electromagnetic lepton pair production have been done for the lead ions at energies $3.4 \mathrm{TeV} /$ nucleon, which yields $\sqrt{s} \approx 6.8 \mathrm{TeV} /$ nucleon. In order to compare the results with our previous work, and also with other authors' work, we will use the energy value at LHC as $3.4 \mathrm{TeV} /$ nucleon as input in our calculations. Therefore, electromagnetic fields at these collisions last a very short time, $\Delta t=b /(\gamma c \beta)$, and the Fourier frequencies could be up to $\omega_{\max } \sim \gamma c \beta / b[11,24]$. We can write the Lagrangian density as the sum of the noninteracting fermion Lagrangian $\mathcal{L}_{0}$ and the Lagrangian density for the coupling of the classical electromagnetic potential to the lepton fields $\mathcal{L}_{\text {int }}[25,26]$,

$$
\begin{aligned}
\mathcal{L}(x) & =\mathcal{L}_{0}(x)+\mathcal{L}_{\text {int }}(x) \\
& =\bar{\Psi}(x)\left[\gamma_{\mu} i \partial^{\mu}-m\right] \Psi(x)-\bar{\Psi}(x) \gamma_{\mu} \Psi(x) A^{\mu}(x),
\end{aligned}
$$

where $A_{1}^{\mu} \equiv\left(A_{1}^{0}, \mathbf{A}_{1}\right)$ is the four-vector potential of the nucleus 1 and the scalar component of the potential can be written as

$$
A_{1}^{0}=-8 \pi^{2} Z \gamma^{2} \delta\left(q_{0}+\beta q_{z}\right) \frac{e^{-i \mathbf{q}_{\perp} \cdot \mathbf{b} / 2}}{\left(q_{z}^{2}+\gamma^{2} q_{\perp}^{2}\right)} G_{E}\left(q^{2}\right) f_{Z}\left(q^{2}\right)
$$

and the non-zero component of the vector potential $A_{1}^{3}$ is in the longitudinal direction and we obtain

$A_{1}^{3}=-\beta A_{1}^{0}$ where the transverse components of the vector potentials are $A_{1}^{1}=A_{1}^{2}=0$. The potentials from nucleus 2 can be written by the substitutions, $\mathbf{b} \rightarrow-\mathbf{b}$ and $\beta \rightarrow-\beta$. In the above equation $f_{Z}\left(q^{2}\right)$ is the form factor of a nucleus which gives the momentum distribution of a proton in the nucleus, and $G_{E}\left(q^{2}\right)$ is the form factor of a proton which represents the electric distribution of the proton $[11,27]$. As we mentioned above, we have used two parameter Fermi $(2 \mathrm{pF})$ function (or Wood-Saxon function) for the charge distribution of the protons:

$\rho(r)=\frac{\rho_{0}}{1+\exp ((r-R) / a)}$

where $R$ is the radius of the nucleus and $a$ is the skin depth or diffuseness parameter. These parameters are obtained by fits to the electron scattering data [28] and $\rho_{0}$ is written by the normalization condition. For symmetric nuclei, the nuclear density for a nucleus that has mass number $A$, a distance $r$ from its center is modeled in the literature with a Woods-Saxon distribution as in Eq. (9). When we normalize the Woods-Saxon charge density to unity we can obtain the parameters: $\rho_{0}=\frac{0.1694}{A} \mathrm{fm}^{-3}$ for the Au nucleus and $\rho_{0}=\frac{0.1604}{A} \mathrm{fm}^{-3}$ for the $\mathrm{Pb}$ nucleus [3]. The radii of the gold and lead nucleus are equal to $R_{\mathrm{Au}}=6.38 \mathrm{fm}$ and $R_{\mathrm{Pb}}=6.62$ $\mathrm{fm}$, respectively. However, recent measurement [7] indicates that the neutrons are differently distributed from the protons, so that the parameters $R$ and $a$ must be modified. Therefore this affects the cross section of the heavy lepton pair production [29]. In order to see the effects of the neutron distributions in the nucleus, we need to have an analytical expression of the Fourier transforms of the Wood-Saxon distribution [30]:

$$
\begin{aligned}
f_{Z}\left(q^{2}\right)= & \int_{0}^{\infty} \frac{4 \pi}{q} \rho(r) \operatorname{Sin}(q r) \mathrm{d} r \\
= & \int_{0}^{\infty} \frac{4 \pi}{q r} \rho(r) \operatorname{Sin}(q r) r^{2} \mathrm{~d} r \\
= & \frac{4 \pi^{2} \rho_{0} a^{3}}{(q a)^{2} \operatorname{Sinh}^{2}(\pi q a)}[\pi q a \operatorname{Cosh}(\pi q a) \operatorname{Sin}(q R) \\
& -q R \operatorname{Cos}(q R) \operatorname{Sinh}(\pi q a)] \\
& +8 \pi \rho_{0} a^{3} \sum_{n=1}^{\infty}(-1)^{n-1} \frac{n e^{-n R / a}}{\left[n^{2}+(q a)^{2}\right]^{2}} .
\end{aligned}
$$

The parameters $R$ and $a$ are explicitly shown in this analytical equation and it is plotted in Fig. 3 together with the numerical result from Fourier transformation of a WoodsSaxon distribution. The agreement is excellent. Since the contribution of the last term in the above equation is very small compared to the first term, it is safely neglected. By changing the parameters $R$ and $a$ we can estimate the sensitivity of the nucleon distributions in the nucleus.

On the other hand, for the electric distribution function of the proton, we can write the dipole form factor for the proton 


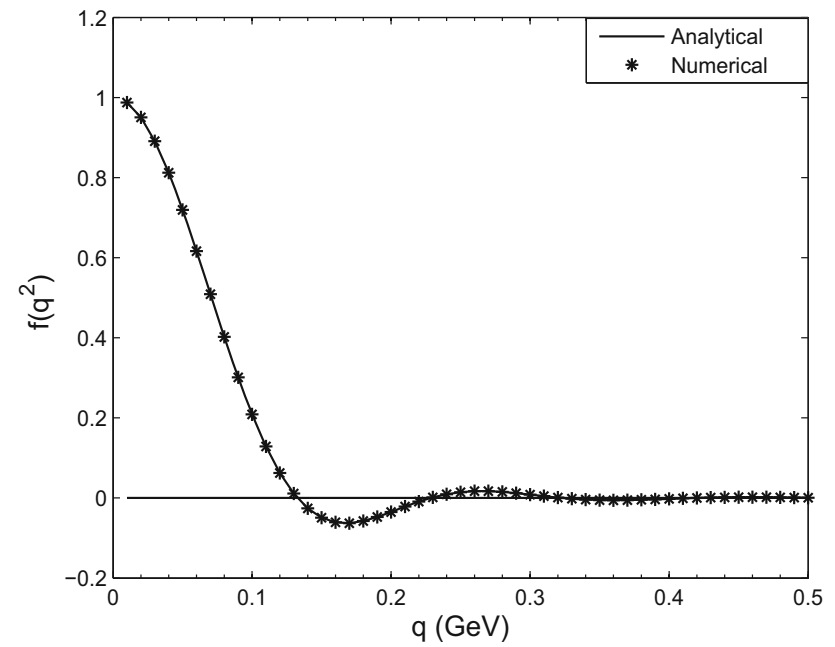

Fig. 3 The electromagnetic form factor for gold. The solid line is the analytic form of the Woods-Saxon distribution and the star line is the numerical calculation of the same equation

as

$$
G_{E}\left(q^{2}\right)=\left(\frac{\Lambda^{2}}{\Lambda^{2}+q^{2}}\right)^{2}
$$

where the value of $\Lambda^{2}$ is $0.71 \mathrm{GeV}^{2}$ [27]. We have observed that the effect of this distribution is negligible. Finally, the total electromagnetic potential is the sum of the nucleus 1 and 2 ,

$A^{\mu}=A_{1}^{\mu}+A_{2}^{\mu}$.

To calculate the total cross section for the heavy-lepton pair production, we first represent the direct term thus:

$$
\begin{aligned}
& \left\langle\Psi_{k}^{(+)}\left|S_{a b}\right| \Psi_{q}^{(-)}\right\rangle \\
& =i \sum_{p} \sum_{s} \int_{-\infty}^{\infty} \frac{d \omega}{2 \pi} \frac{\left\langle\Psi_{k}^{(+)}\left|V_{a}\left(\omega-E_{k}^{(+)}\right)\right| \chi_{p}^{(s)}\right\rangle\left\langle\chi_{p}^{(s)}\left|V_{b}\left(E_{q}^{(-)}-\omega\right)\right| \Psi_{q}^{(-)}\right\rangle}{\left(E_{p}^{(s)}-\omega\right)} \\
& =i \sum_{p} \sum_{s} \int_{-\infty}^{\infty} \frac{d \omega}{2 \pi} \int_{-\infty}^{\infty} d^{3} \mathbf{r} e^{-i(\mathbf{k}-\mathbf{p}) \cdot \mathbf{r}} A_{a}\left(\mathbf{r} ; \omega-E_{k}^{(+)}\right) \\
& \quad \times \int_{-\infty}^{\infty} d^{3} \mathbf{r}^{\prime} e^{-i(\mathbf{p}-\mathbf{q}) \cdot \mathbf{r}^{\prime}} A_{b}\left(\mathbf{r}^{\prime} ; E_{q}^{(-)}-\omega\right) \\
& \quad \times \frac{\left\langle\mathbf{u}_{\sigma_{k}}^{(+)}\left|\left(1-\beta \alpha_{z}\right)\right| \mathbf{u}_{\sigma_{p}}^{(s)}\right\rangle\left\langle\mathbf{u}_{\sigma_{p}}^{(s)}\left|\left(1+\beta \alpha_{z}\right)\right| \mathbf{u}_{\sigma_{q}}^{(-)}\right\rangle}{\left(E_{p}^{(s)}-\omega\right)}
\end{aligned}
$$

Thus, by combining both integrals we obtain for the direct free-free pair production amplitude the explicit expression:

$$
\begin{aligned}
& \left\langle\Psi_{k}^{(+)}\left|S_{a b}\right| \Psi_{q}^{(-)}\right\rangle \\
& =i \sum_{s} \sum_{\sigma_{p}} \int \frac{d^{3} \mathbf{p}}{(2 \pi)^{3}} \int \frac{d \omega}{2 \pi} e^{i\left[\mathbf{p}_{\perp}-\left(\frac{\mathbf{k}_{\perp}+\mathbf{q}_{\perp}}{2}\right)\right] \cdot \mathbf{b}} 8 \pi^{2} Z \gamma^{2} \\
& \quad \times \frac{\delta\left(\omega-E_{k}^{(+)}+\beta\left(k_{z}-p_{z}\right)\right)}{\left(k_{z}-p_{z}\right)^{2}+\gamma^{2}\left(\mathbf{k}_{\perp}-\mathbf{p}_{\perp}\right)^{2}}
\end{aligned}
$$

$$
\begin{aligned}
& \times 8 \pi^{2} Z \gamma^{2} \frac{\delta\left(E_{q}^{(-)}-\omega-\beta\left(p_{z}-q_{z}\right)\right)}{\left(p_{z}-q_{z}\right)^{2}+\gamma^{2}\left(\mathbf{p}_{\perp}-\mathbf{q}_{\perp}\right)^{2}} \\
& \times \frac{\left\langle\mathbf{u}_{\sigma_{k}}^{(+)}\left|\left(1-\beta \alpha_{z}\right)\right| \mathbf{u}_{\sigma_{p}}^{(s)}\right\rangle\left\langle\mathbf{u}_{\sigma_{p}}^{(s)}\left|\left(1+\beta \alpha_{z}\right)\right| \mathbf{u}_{\sigma_{q}}^{(-)}\right\rangle}{E_{p}^{(s)}-\omega},
\end{aligned}
$$

$E_{p}^{(s)}$ is the energy of the intermediate state. In these equations $\mathbf{k}$ represents the momentum of the electron and $\mathbf{q}$ represents the momentum of the positron. The vector $\mathbf{p}$ describes the momentum of the intermediate (electron and positron) states in the field of the ion and it can be decomposed into its transverse and parallel part, $\mathbf{p}=\mathbf{p}_{\perp}+p_{z}$, relative to the motion of the ions. The momentum that is parallel to the heavy ions and the frequency in Eq. (13) are fixed by momentum conservation;

$p_{z}=\frac{E_{q}^{(-)}-E_{k}^{(+)}+\beta\left(k_{z}+q_{z}\right)}{2 \beta}$,

$\omega=\frac{E_{k}^{(+)}+E_{q}^{(-)}-\beta\left(k_{z}-q_{z}\right)}{2}$.

For the fixed momentum and the spin states, the transition matrix element can be written as

$$
\begin{aligned}
\left\langle\Psi_{k}^{(+)}\left|S_{a b}\right| \Psi_{q}^{(-)}\right\rangle & \\
= & \frac{i}{2 \beta} \int \frac{\mathrm{d}^{2} p_{\perp}}{(2 \pi)^{2}} e^{i\left[\mathbf{p}_{\perp}-\left(\frac{\mathbf{k}_{\perp}+\mathbf{q}_{\perp}}{2}\right)\right] \cdot \mathbf{b}} \\
& \times F\left(\mathbf{k}_{\perp}-\mathbf{p}_{\perp}: \omega_{a}\right) F\left(\mathbf{p}_{\perp}-\mathbf{q}_{\perp}: \omega_{b}\right) \mathcal{T}_{k q}\left(\mathbf{p}_{\perp}:+\beta\right),
\end{aligned}
$$

$\omega_{a}$ and $\omega_{b}$ are the frequencies associated with the fields of ions $a$ and $b$, respectively,

$\omega_{a}=\frac{E_{q}^{(-)}-E_{k}^{(+)}+\beta\left(q_{z}-k_{z}\right)}{2}$,

$\omega_{b}=\frac{E_{q}^{(-)}-E_{k}^{(+)}-\beta\left(q_{z}-k_{z}\right)}{2}$,

the function $F$ is the scalar part of the field from each heavy ion,

$$
F\left(\mathbf{k}_{\perp}-\mathbf{p}_{\perp}: \omega_{a}\right)=\frac{4 \pi Z \gamma^{2} \beta^{2}}{\left(\omega_{a}^{2}+\gamma^{2} \beta^{2}\left(\mathbf{k}_{\perp}-\mathbf{p}_{\perp}\right)^{2}\right)},
$$

for the frequency $\omega_{a}$, and

$$
F\left(\mathbf{p}_{\perp}-\mathbf{q}_{\perp}: \omega_{b}\right)=\frac{4 \pi Z e \gamma^{2} \beta^{2}}{\left(\omega_{b}^{2}+\gamma^{2} \beta^{2}\left(\mathbf{p}_{\perp}-\mathbf{q}_{\perp}\right)^{2}\right)},
$$

for the frequency $\omega_{b}$, respectively. The function $\mathcal{T}$ explicitly depends on the velocity of the heavy ions $\beta$, on the transverse momentum $\mathbf{p}_{\perp}$, and on the states $k, q$; 


$$
\begin{aligned}
& \mathcal{T}_{k q}\left(\mathbf{p}_{\perp}:+\beta\right) \\
& =\sum_{s} \sum_{\sigma_{p}} \frac{1}{\left(E_{p}^{(s)}-\left(\frac{E_{k}^{(+)}+E_{q}^{(-)}}{2}\right)+\beta\left(\frac{k_{z}-q_{z}}{2}\right)\right)} \\
& \quad \times\left\langle\mathbf{u}_{\sigma_{k}}^{(+)}\left|\left(1-\beta \alpha_{z}\right)\right| \mathbf{u}_{\sigma_{p}}^{(s)}\right\rangle\left\langle\mathbf{u}_{\sigma_{p}}^{(s)}\left|\left(1+\beta \alpha_{z}\right)\right| \mathbf{u}_{\sigma_{q}}^{(-)}\right\rangle .
\end{aligned}
$$

Having the amplitudes for the direct and crossed diagram, we are now prepared to write down the cross section for the generation of a free-free pair in collisions of two heavy ions

$\sigma=\int d^{2} b \sum_{k>0} \sum_{q<0}\left|\left\langle\Psi_{k}^{(+)}|S| \Psi_{q}^{(-)}\right\rangle\right|^{2}$,

where $S=S_{a b}+S_{b a}$ denotes the sum of the direct and crossed cases. Making use of all the simplifications from above, these cross sections can be expressed as

$$
\begin{aligned}
\sigma= & \int d^{2} b \sum_{k>0} \sum_{q<0}\left|\left\langle\Psi_{k}^{(+)}\left|S_{a b}\right| \Psi_{q}^{(-)}\right\rangle+\left\langle\Psi_{k}^{(+)}\left|S_{b a}\right| \Psi_{q}^{(-)}\right\rangle\right|^{2} \\
= & \sum_{\sigma_{k}} \sum_{\sigma_{q}} \int \frac{d^{3} k d^{3} q d^{2} p_{\perp}}{(2 \pi)^{8}} \\
& \left.\times\left(\mathcal{A}^{(+)}\left(k, q ; \mathbf{p}_{\perp}\right)+\mathcal{A}^{(-)}\left(k, q ; \mathbf{p}_{\perp}\right)\right)\right)^{2},
\end{aligned}
$$

with

$$
\begin{aligned}
\mathcal{A}^{(+)}\left(k, q ; \mathbf{p}_{\perp}\right)= & F\left(\mathbf{k}_{\perp}-\mathbf{p}_{\perp}: \omega_{a}\right) F\left(\mathbf{p}_{\perp}-\mathbf{q}_{\perp}: \omega_{b}\right) \\
& \mathcal{T}_{k q}\left(\mathbf{p}_{\perp}:+\beta\right),
\end{aligned}
$$

and

$$
\begin{aligned}
\mathcal{A}^{(-)}\left(k, q ; \mathbf{p}_{\perp}\right)= & F\left(\mathbf{k}_{\perp}-\mathbf{p}_{\perp}: \omega_{b}\right) F\left(\mathbf{p}_{\perp}-\mathbf{q}_{\perp}: \omega_{a}\right) \\
& \mathcal{T}_{k q}\left(\mathbf{p}_{\perp}:-\beta\right),
\end{aligned}
$$

these being some proper products of the transition amplitudes and scalar parts of the fields as associated with ions $a$ and $b$.

\section{Calculations and tables}

Table 1 displays the cross sections of electromagnetic productions of electron, muon, and tauon pairs from the ultrarelativistic gold-gold collisions at RHIC energies and leadlead collisions at LHC energies with no form factor and with a Wood-Saxon form factor distribution. In this calculation, we simply used the analytical expression of the Fourier transformation of this form factor. We have also included the electric distribution function of the proton (Eq. (11)) in our calculation, and we have seen that it has a negligible effect for the production of any lepton pairs. The cross sections of electromagnetic productions of electron, muon, and tauon pairs are also calculated in [31] by using various form factors such as uniform, Gaussian, and Woods-Saxon types, and we compared the results between them. In this present work, we are
Table 1 The cross sections of electromagnetic productions of electron-, muon-, and tauon-pair productions from the ultra-relativistic gold-gold collisions at RHIC energies and lead-lead collisions at LHC energies. $f^{\mathrm{NFF}}$ means the form factor is not included and $f^{W S}$ means that the form factor is included in the calculations

\begin{tabular}{cll}
\hline & $\sigma^{f^{\mathrm{NFF}}}($ barn $)$ & $\sigma^{f^{\mathrm{WS}}}($ barn $)$ \\
\hline$e^{-} e^{+}$ & & \\
RHIC & $3.63 \times 10^{4}$ & $3.62 \times 10^{4}$ \\
LHC & $2.44 \times 10^{5}$ & $2.43 \times 10^{5}$ \\
$\mu^{-} \mu^{+}$ & & \\
RHIC & $7.3 \times 10^{-1}$ & $2.03 \times 10^{-1}$ \\
LHC & 5.2 & 2.7 \\
$\tau^{-} \tau^{+}$ & & \\
RHIC & $4.7 \times 10^{-4}$ & $3.9 \times 10^{-6}$ \\
LHC & $7.7 \times 10^{-3}$ & $1.6 \times 10^{-3}$ \\
\hline
\end{tabular}

using the Woods-Saxon type form factor since it is more realistic and widely used in the literature.

When we compare electron pair production cross sections with and without form factor, it is clearly seen that the differences between the results are negligibly small. Since the Compton wavelength of the electron is much larger than the radius of the colliding heavy ions, the form factors of the proton and nucleus almost have no effect for the production of electron-positron pairs.

However, when we compare muon- and tauon-pair production cross sections with and without form factors, it is clearly seen that the differences are large. The cross section results for muon- and tauon-pair production with form factors are much smaller than without form factors. Since the Compton wavelengths of muons and tauons are smaller than the radius of the colliding heavy ions, the effects of the form factors become dominant for pair production. At RHIC and LHC energies, muon-pair production is reduced by factors of about 3 and 2, respectively. On the other hand, at RHIC and LHC energies, tauon-pair production is reduced by factors of about 100 and 5 , respectively.

Figures 4, 5, and 6 display the differential cross sections as a function of the energy $p_{0}$, the longitudinal momentum $p_{z}$, the transverse momentum $p_{\perp}$, and the rapidity $y$ of the produced electron, muon, and tauon for the cases with and without form factors at RHIC and LHC energies. When we compare these figures, in Fig. 4 for the electron pairs, it is clearly seen that the form factor has almost no effect on the cross section. With and without form factors, differential cross sections show the same behavior and almost identical values. The differential cross section as a function of transverse momentum decreases rapidly compared to the longitudinal momentum and energy. The differential cross sections as a function longitudinal momentum and energy have almost identical values. This also shows that the produced leptons 


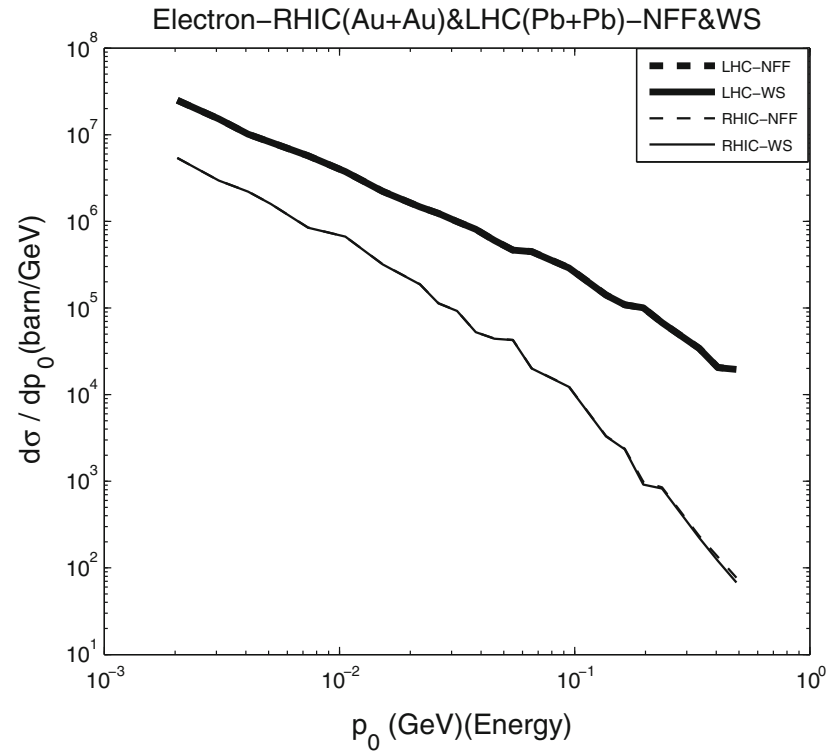

(a)

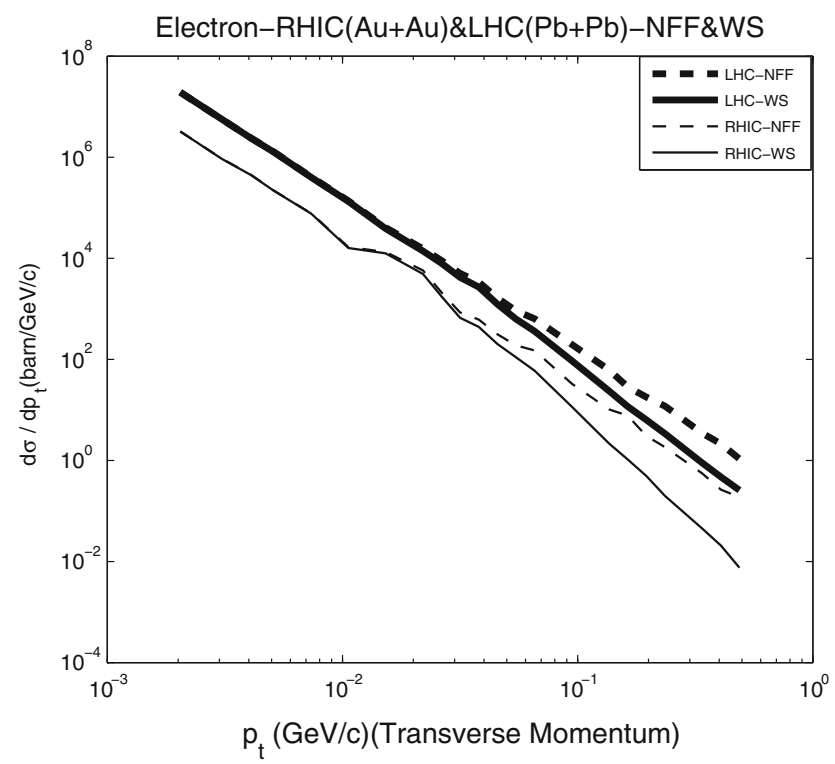

(c)

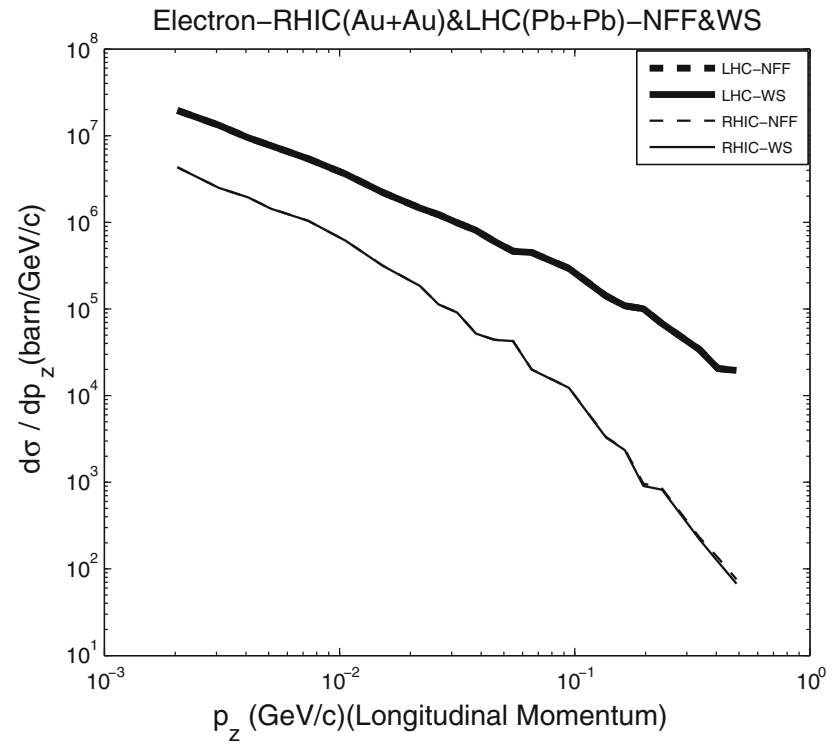

(b)

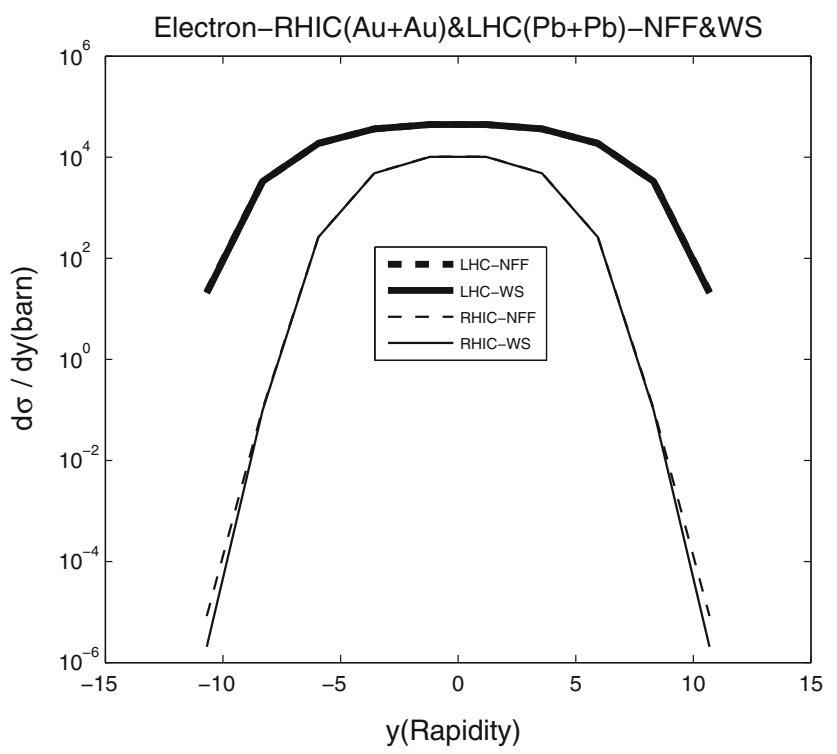

(d)

Fig. 4 The differential cross section as a function of a energy $\left(p_{0}\right)$, b longitudinal momentum $\left(p_{z}\right)$, $\mathbf{c}$ transverse momentum $\left(p_{\perp}\right)$ and $\mathbf{d}$ rapidity $(y)$ of the produced electron at RHIC and LHC energies with and without form factors

carry energy in the longitudinal direction where the heavy ions move.

On the other hand, In Figs. 5 and 6 the same differential cross sections of the produced muon and tauon pairs at RHIC and LHC energies with and without form factors are shown. When we compare these figures, it is clear that the longitudinal momentum of the produced muon and tauon are much higher than the transverse momentum. Therefore, the energy $E=\sqrt{p_{t}^{2}+p_{z}^{2}+1}$ is carried by the longitudinal momentum of the produced heavy leptons. It is also clear that the differential cross sections as a function of energy and longitudinal momentum are very close to each other at RHIC and LHC energies. The rapidity distribution of the muonpair production and form factor reduce the cross sections by about ten times at the RHIC energies, and they reduce the cross section by about five times at the LHC energies. For the tauon-pair production, the reduction of the cross section is about two orders of magnitude for the RHIC energies, and it is about one order of magnitude for the LHC energies. All these calculations show that distributions of 


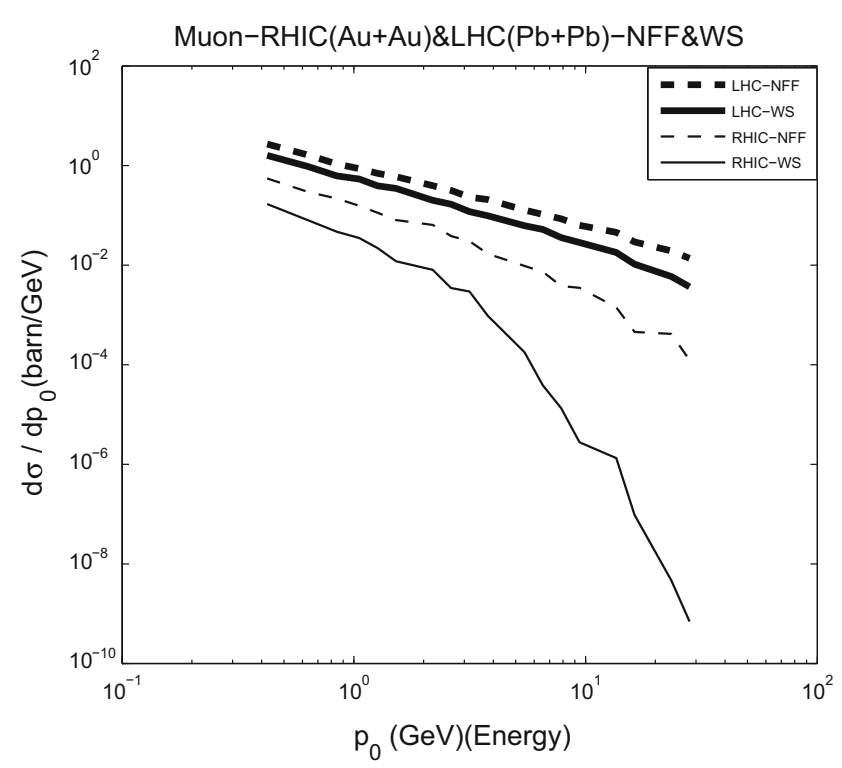

(a)

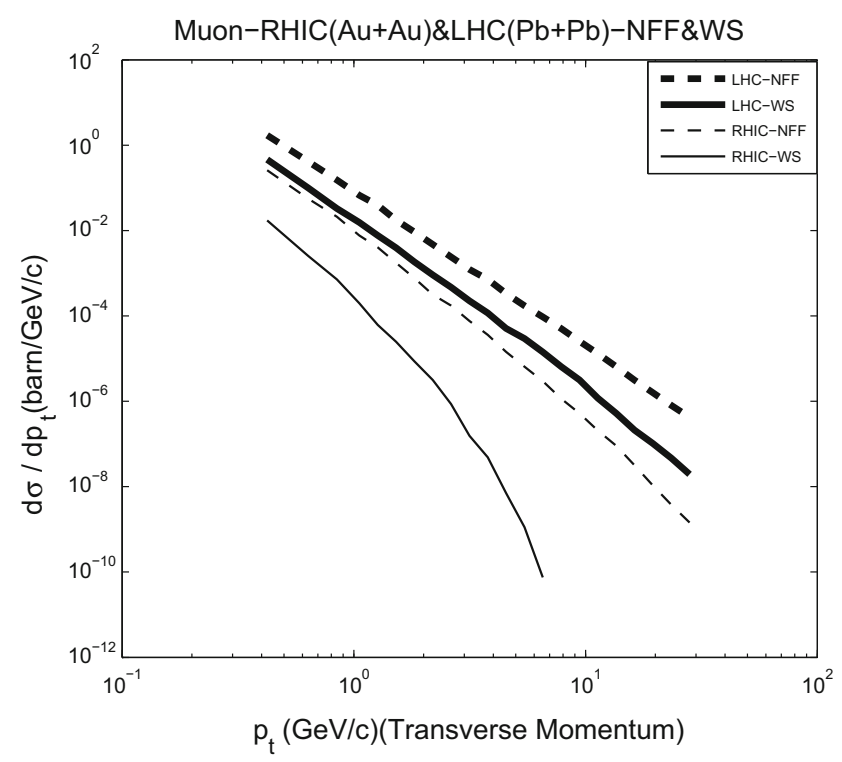

(c)

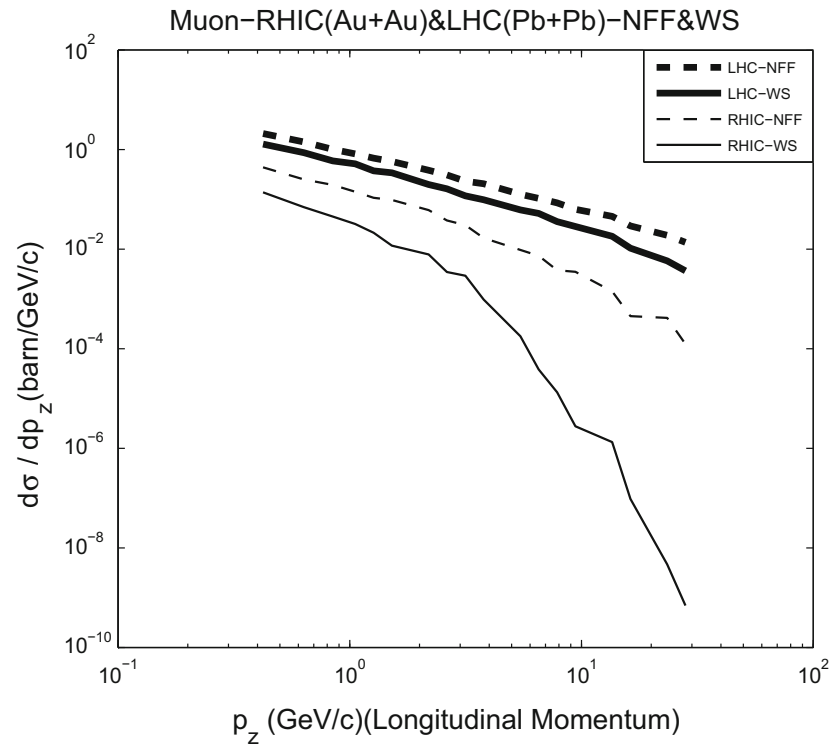

(b)

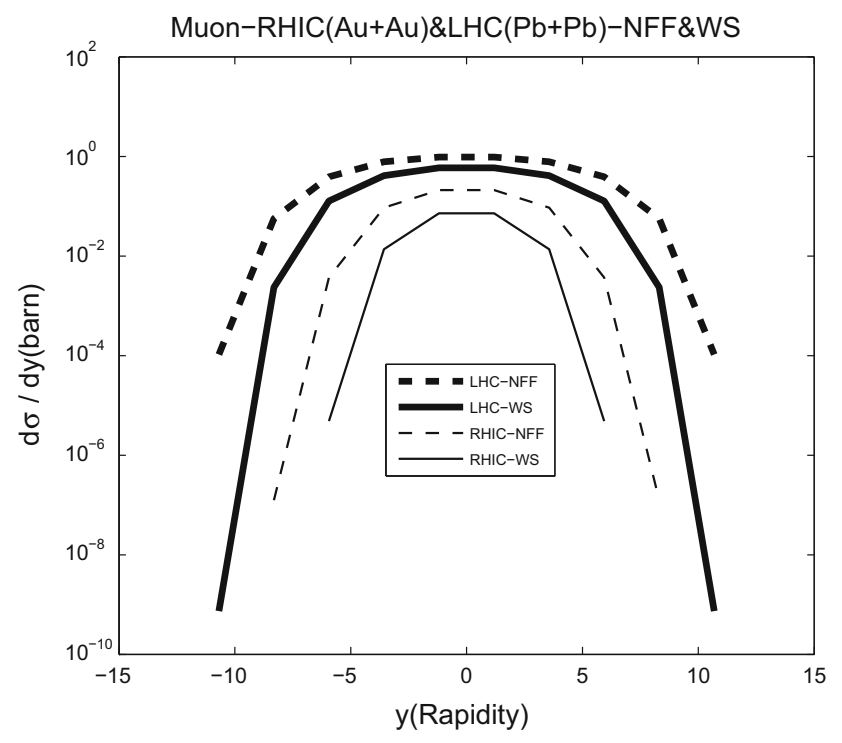

(d)

Fig. 5 The differential cross section as a function of a energy $\left(p_{0}\right)$, b longitudinal momentum $\left(p_{z}\right)$, c transverse momentum $\left(p_{\perp}\right)$ and $\mathbf{d}$ rapidity ( $y$ ) of the produced muon at RHIC and LHC energies with and without form factors

the heavy leptons are mainly confined to the central rapidity range.

Figure 7 displays muon and tauon total cross sections for $\mathrm{Au}+\mathrm{Au}$ and $\mathrm{Pb}+\mathrm{Pb}$ collisions as a function of the Lorentz factor $\gamma$ of the beams for the Wood-Saxon form factors. We also compare this results with the case of no form factor. For the muon-pair production, the total cross sections are reduced by factors of about 10 for the low energies, about 3 for the RHIC energies, and about 2 for the LHC energies. For the tauon-pair production, the total cross sections are reduced by factors of about $10^{9}-10^{6}$ for the low energies, about 100 for the RHIC energies, and finally about 5 for the LHC energies.

Figure 8 shows the total cross section of heavy-lepton pair production as a function of the charge of the colliding nuclei $Z$. Obviously the nuclear form factor reduces the total cross sections. At RHIC energies, the form factor reduces the tauon-pair production cross section by about one or two orders of magnitude, however, for the muon-pair production the reduction is about a factor of 2 or 3 . On the other hand, at LHC energies, the form factors reduce the muon-pair production cross section by factors of about 1 or 2 , for the tauon 


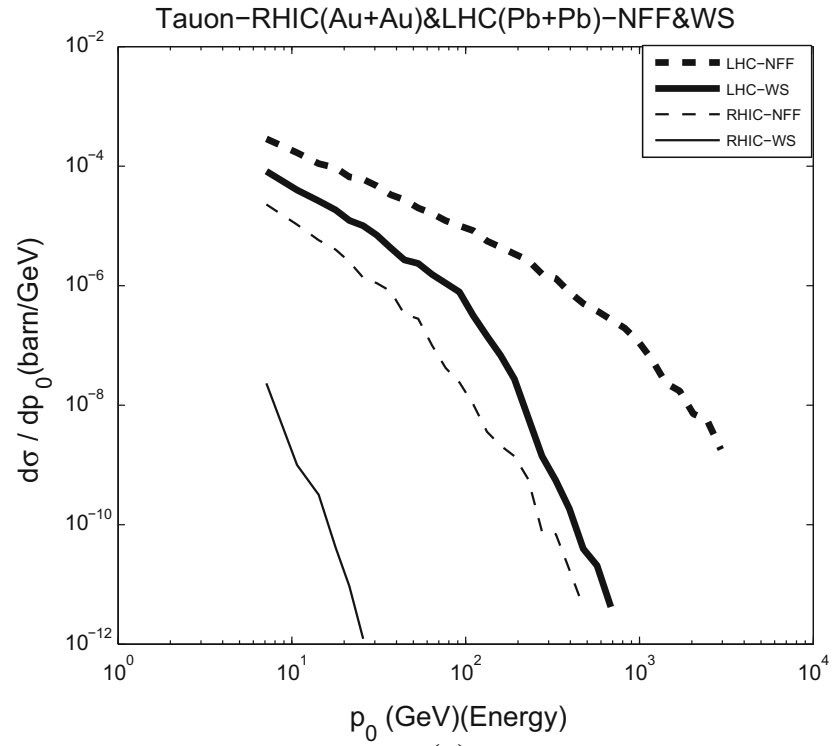

(a)

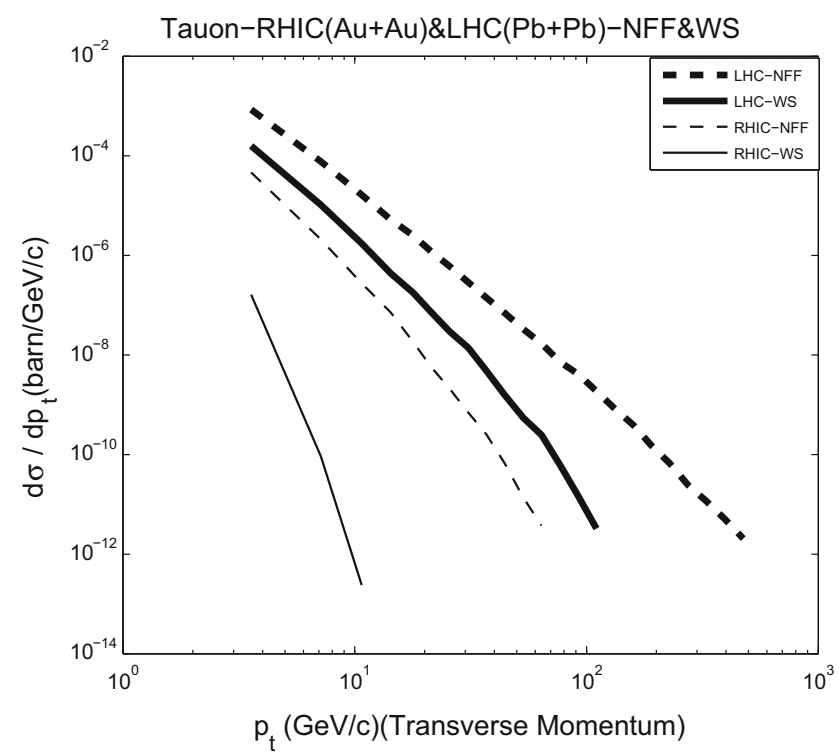

(c)

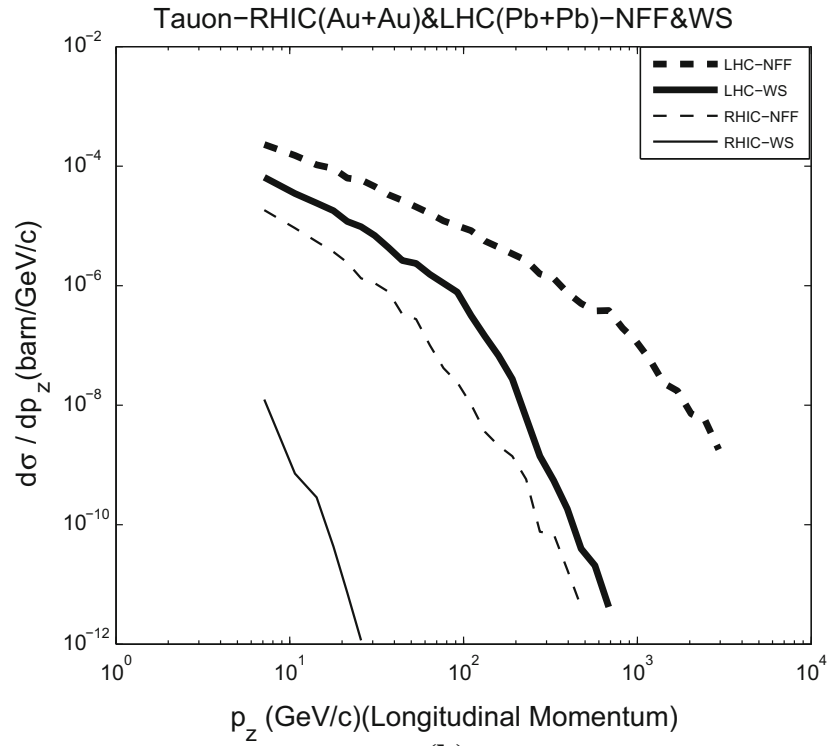

(b)

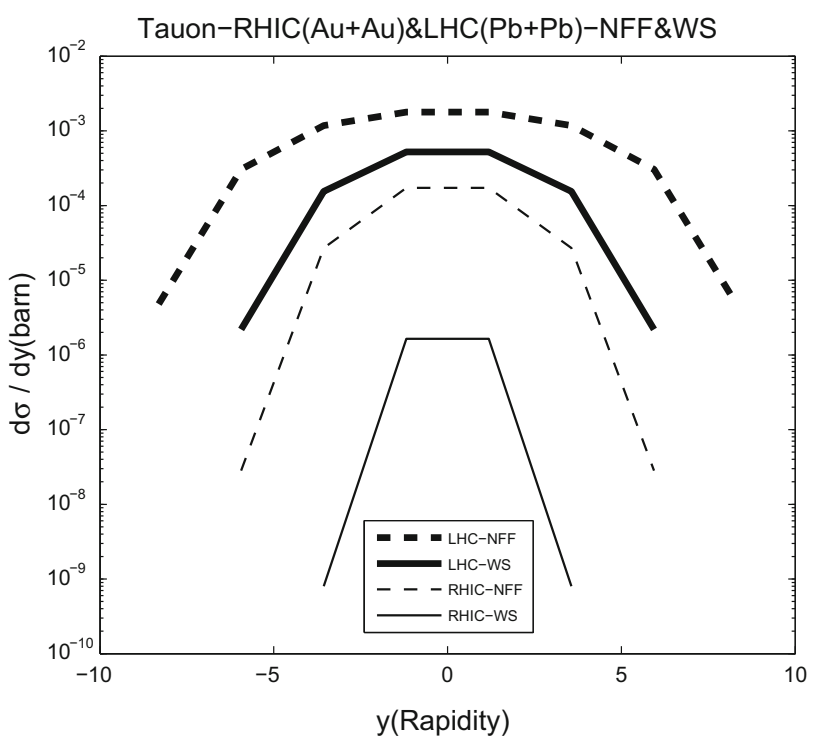

(d)

Fig. 6 The differential cross section as a function of a energy $\left(p_{0}\right)$, b longitudinal momentum $\left(p_{z}\right)$, $\mathbf{c}$ transverse momentum $\left(p_{\perp}\right)$ and $\mathbf{d}$ rapidity $(y)$ of the produced tauon at RHIC and LHC energies with and without form factors

production cross section this reduction is slightly less than one order of magnitude.

Figure 9 displays the impact parameter dependence cross section for muon- and tauon-pair production at RHIC and LHC energies with and without Wood-Saxon distribution. In these calculations, the range of the impact parameter $b$ is between 0 and infinity. Since the Compton wavelengths of the muon are $1.86 \mathrm{fm}$ and for the tauon $0.11 \mathrm{fm}$, which are smaller than the radius of the nucleus, the heavy leptons are produced mainly near the radius of the heavy ions. Maximum production of muon pairs occurs at RHIC ener- gies about impact parameters of the $20 \mathrm{fm}$ range, and at LHC energies in the impact parameter of the 40-80 fm range. At a range of about $200 \mathrm{fm}$, the impact parameter dependence cross section decreases by factors of about 10 at RHIC energies, and 2 at LHC energies. On the other hand, maximum production of tauon pairs occurs for impact parameters of the $7 \mathrm{fm}$ range at RHIC energies, and about $10 \mathrm{fm}$ at LHC energies. At about $200 \mathrm{fm}$, the impact parameter dependence of the cross section decreases by a factor of about 100 at RHIC energies and 50 at LHC energies. 


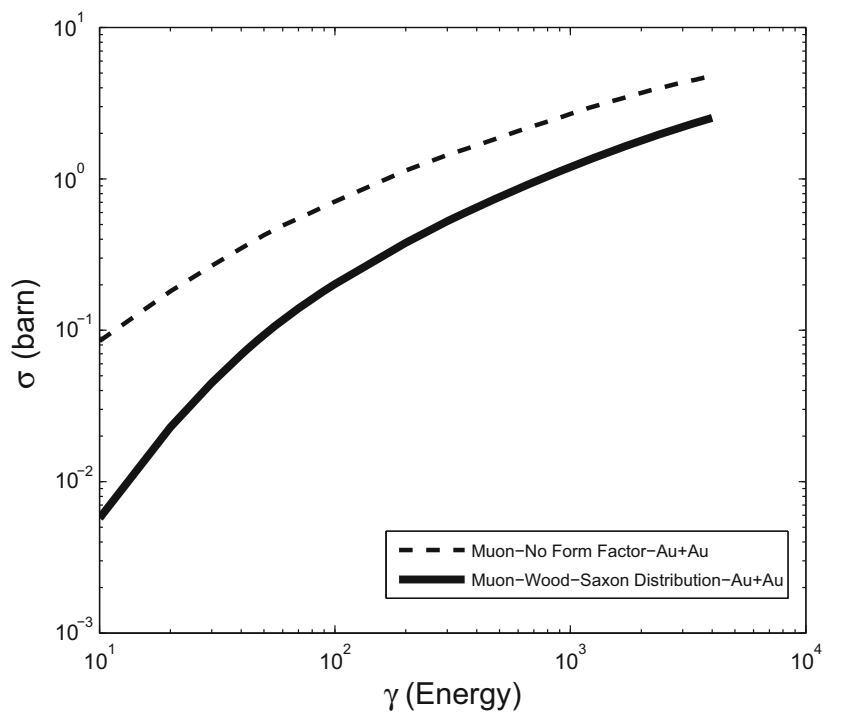

(a)

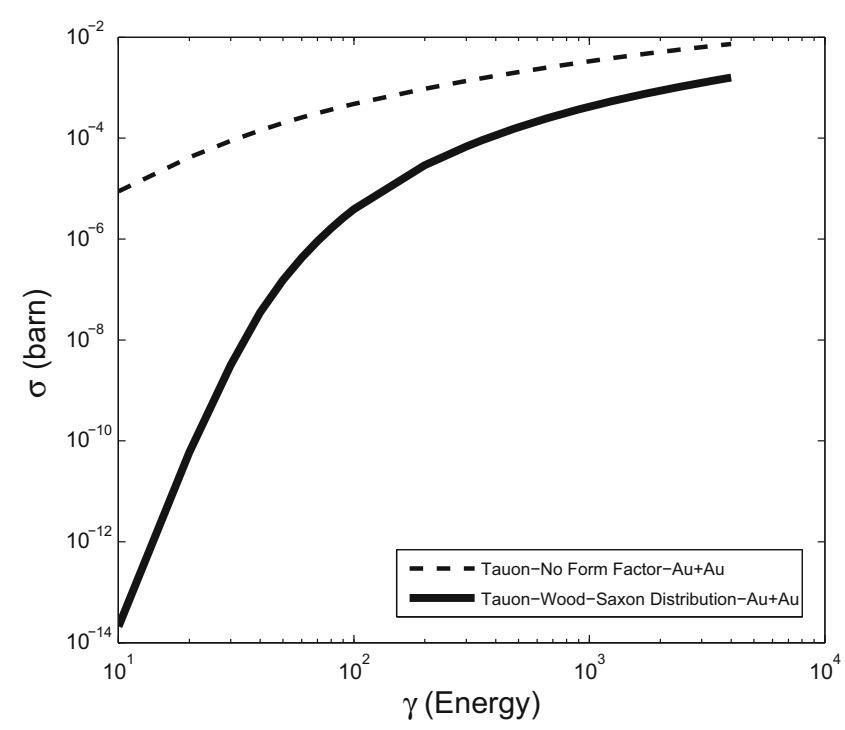

(c)

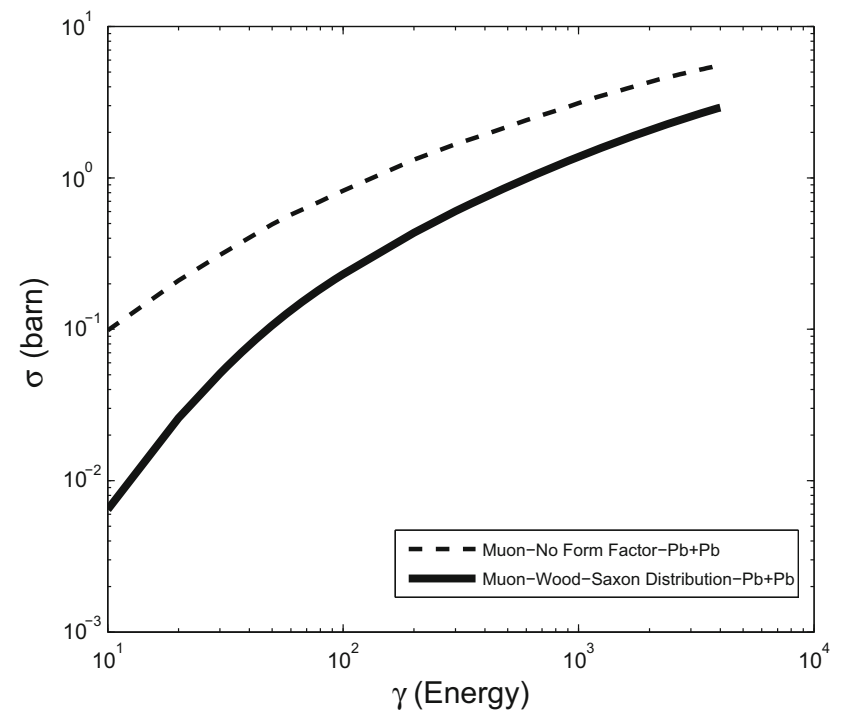

(b)

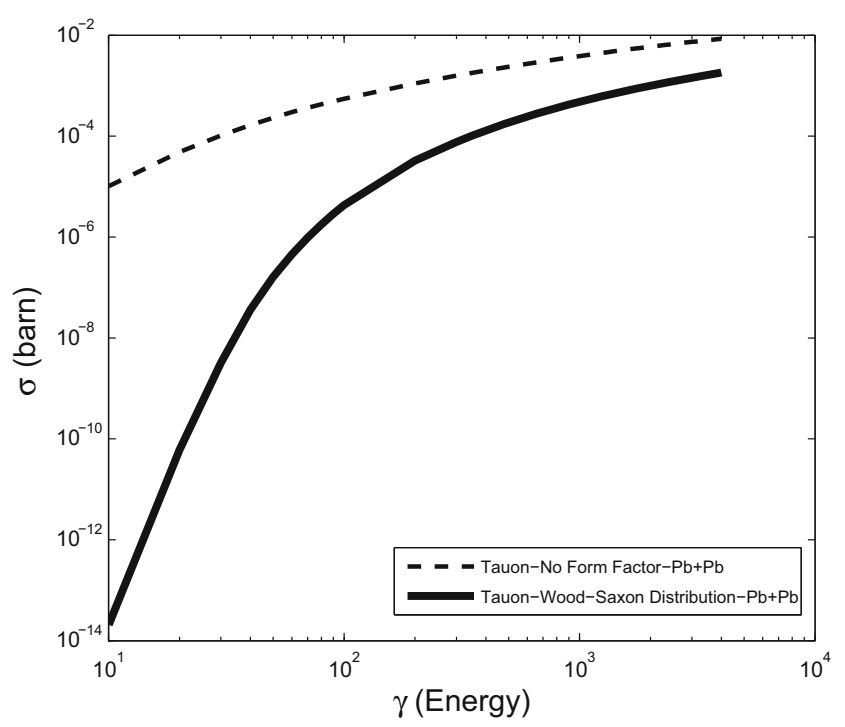

(d)

Fig. 7 Muon and tauon cross sections for $\mathrm{Au}+\mathrm{Au}$ and $\mathrm{Pb}+\mathrm{Pb}$ collisions as a function of the $\gamma$ with and without form factors

Table 2 represents the percentage of cross section contributions to the total cross section between the impact parameter ranges $b<R_{1}+R_{2}$ and $b>R_{1}+R_{2}$ where $R_{1}+R_{2} \sim 15 \mathrm{fm}$ for the heavy ions. The cross section calculations are taken from Table 1 with form factor. For the electron-positron pair production cross section, pairs are overwhelmingly produced for the range of $b>R_{1}+R_{2}$. For the muon- and tauon-pair production, the range $b<R_{1}+R_{2}$ has an important contribution to the total cross sections. When the energy of the colliding ions increases, the contributions from the $b<R_{1}+R_{2}$ range decrease. However, when the mass of the lepton increases, the contribution from the $b<R_{1}+R_{2}$ range increases. We have repeated the cal- culations for the impact parameters $b$ for the ranges 20, 25, and $30 \mathrm{fm}$. These calculations clearly indicate that the details of the form factors, such as the distributions of the nucleons in the nucleus, are important for the calculations of the cross sections.

In all the above calculations, we have also used the proton form factor $G_{E}\left(q^{2}\right)$. Our results show that the contribution of this proton form factor is negligible.

The description of the matter distribution in nuclei is still under investigation in nuclear physics and precise knowledge of this distribution can help us to understand the structure of the nucleus. The charge distribution has been measured with high accuracy from the electron scattering data, so that the 


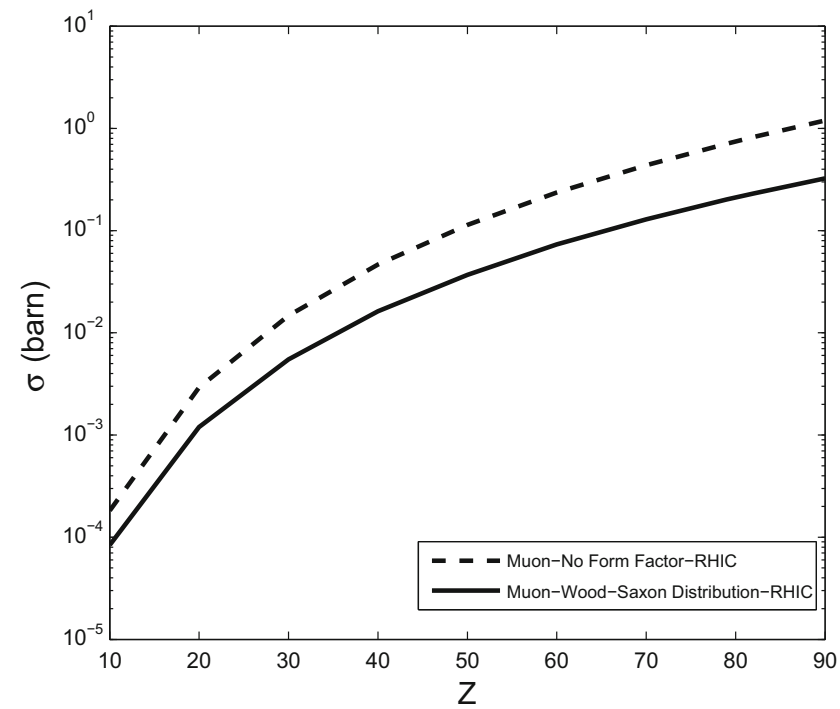

(a)

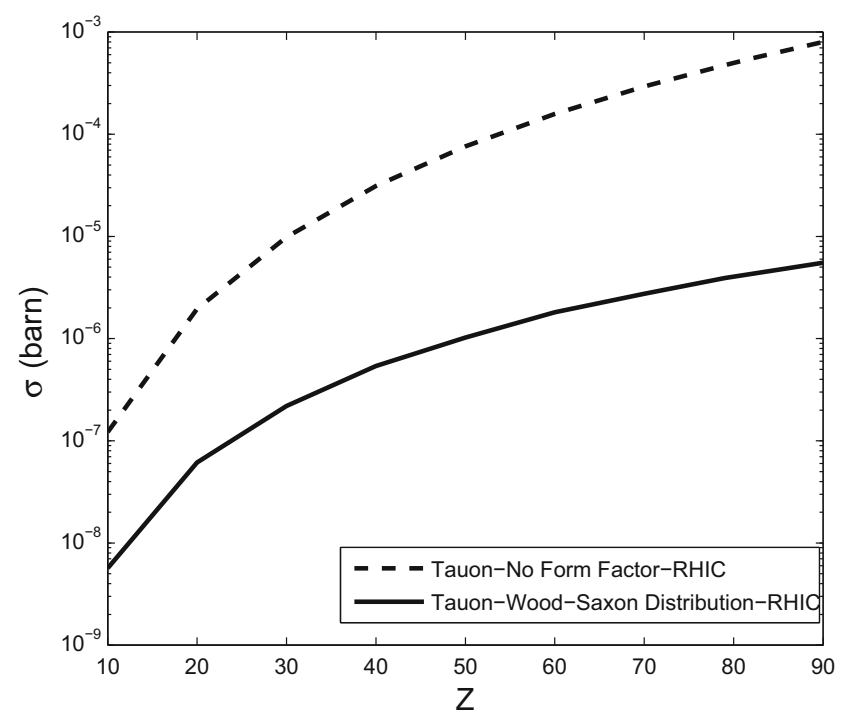

(c)

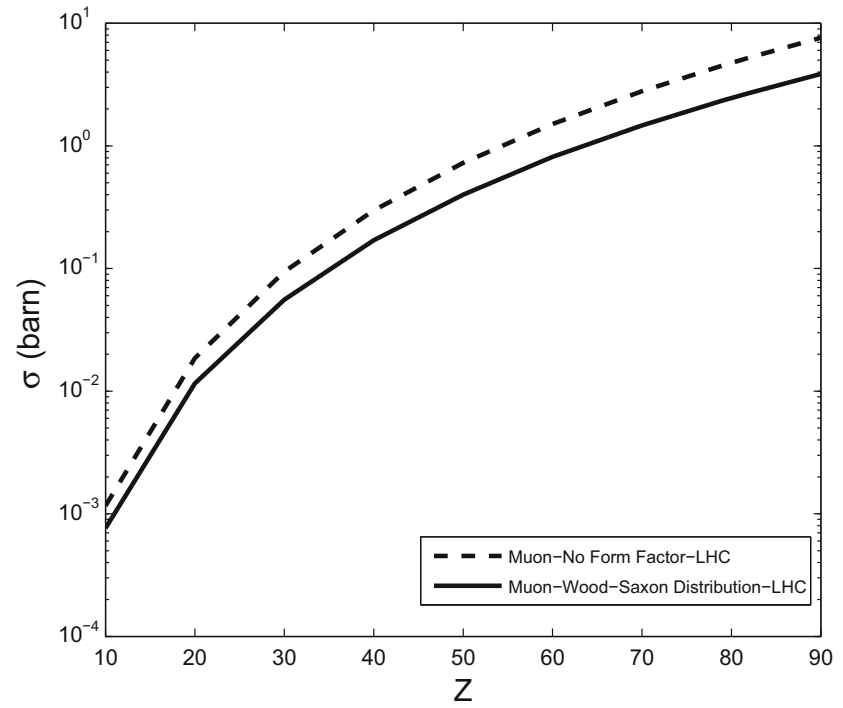

(b)

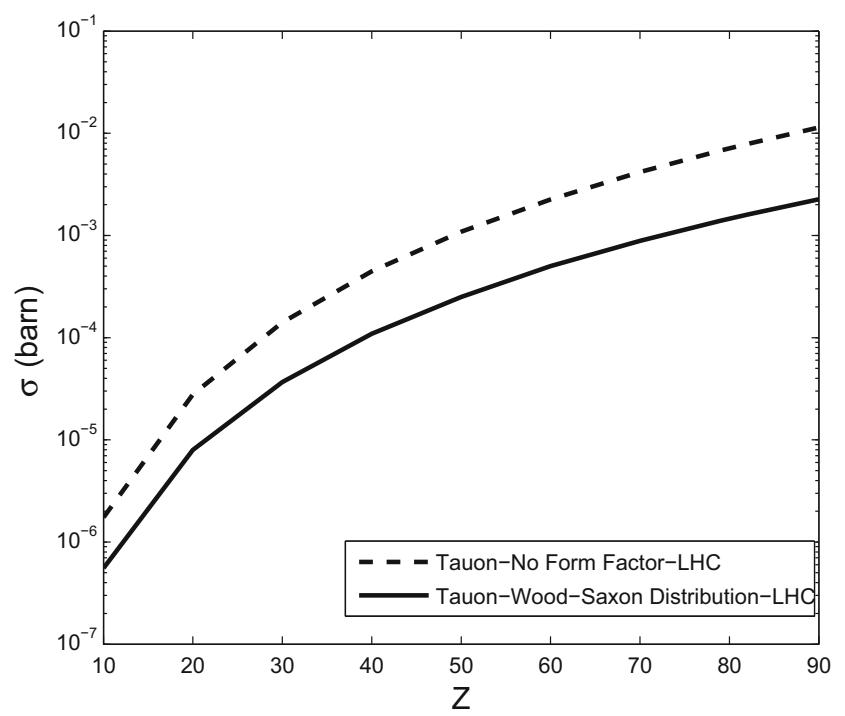

(d)

Fig. 8 Muon and tauon cross sections for RHIC and LHC energies as functions of the nuclear charge Z with and without form factors

charge radii are usually known with uncertainties lower than $1 \%[1,7]$. However, the neutron distribution in the nucleus is not accurately measured as for protons.

We have also investigated the neutron distribution of the nucleus. The Wood-Saxon equation gives us the proton charge distribution and neutron density distribution of the nucleus. In Eq. (8), the parameter $R$ is the radius of the nucleus and $a$ is the skin depth and these parameters are obtained from the electron scattering data [28]. Recent experiments show that the neutrons are distributed differently from the protons and this affects the cross sections of the heavylepton pair productions. Neutrons are electrically neutral, so they do not contribute to the electric form factor. Neutrons can contribute to the hadronic radius. In order to see the effects of the neutron distribution, we have changed the parameters $R$ and $a$ in the Wood-Saxon form factor. First we keep the $R$ constant and change the parameter $a$ between $0.3 \mathrm{fm} \leq a \leq 0.8 \mathrm{fm}$. Although the parameter $a$ has a value of about $0.55 \mathrm{fm}$, by changing it for the extreme ranges, we can see how it affects the cross sections. Next we keep the parameter $a=0.55 \mathrm{fm}$ constant and the change the radius $R$ between $5.8 \mathrm{fm} \leq R \leq 7.2 \mathrm{fm}$. In Figs. 10 and 11, we have plotted these cross sections for muon and tauon productions at RHIC (gold nucleus) and at LHC (lead nucleus). When the parameter $a$ is changed from the value of $0.4 \mathrm{fm}$ to $0.6 \mathrm{fm}$, the cross section $(\Delta \sigma / \sigma)$ of the produced muon pairs decreases by about $4 \%$ at RHIC and $2 \%$ percent at LHC. Similarly, tauon production decreases by about $4 \%$ at LHC and $14 \%$ 


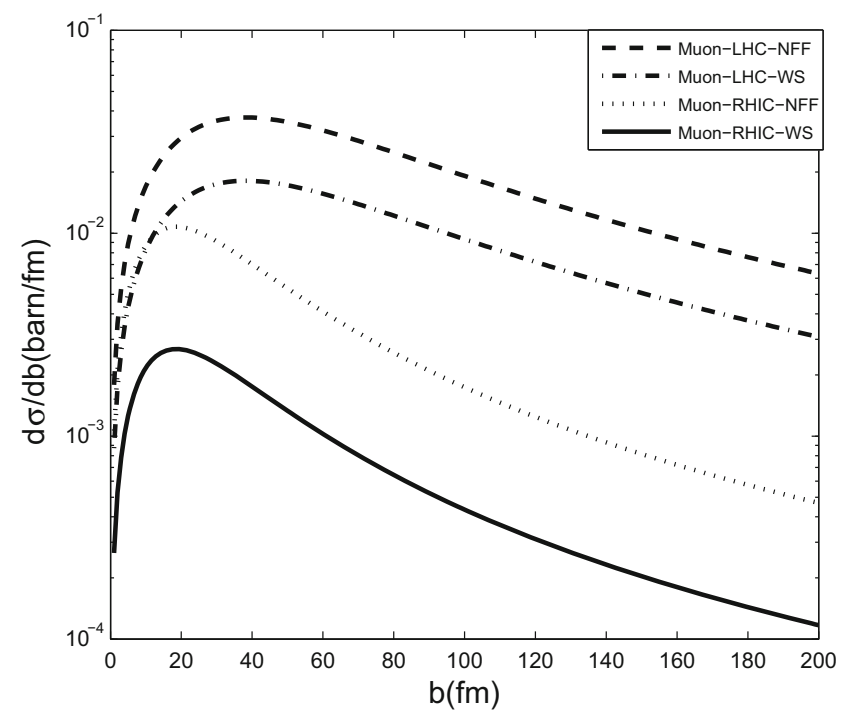

(a)

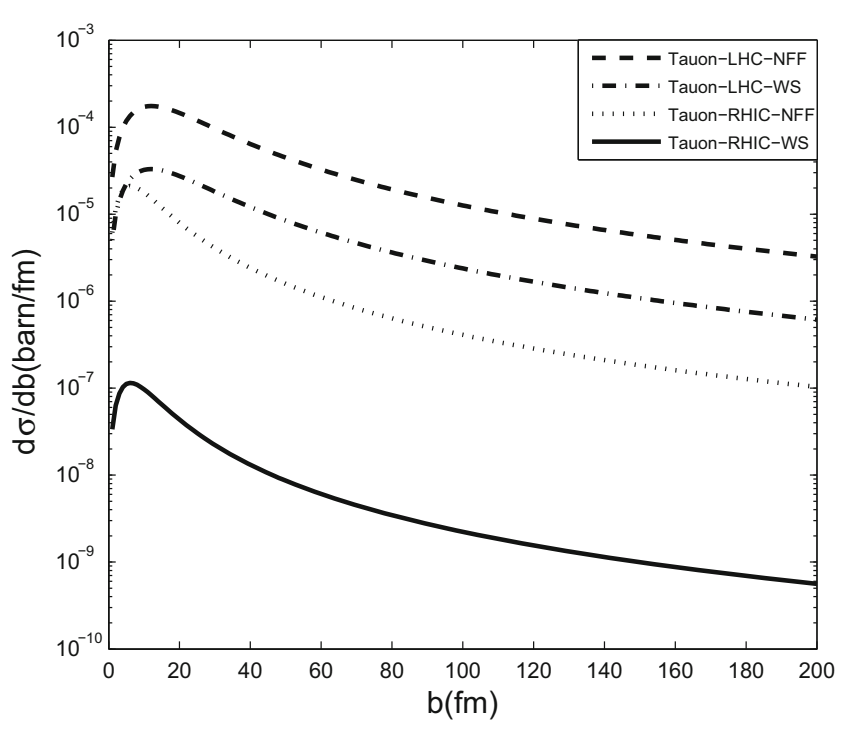

(b)

Fig. 9 Impact parameter dependence cross section for muon and tauon pairs at RHIC and LHC energies with Wood-Saxon distribution and no form factor

Table 2 The percentage of cross section contributions to the total cross section between the impact parameter ranges $0<b<15-20-25-30 \mathrm{fm}$ and 15-20-25-30 fm $<b<\infty$.

The cross section calculations are taken from Table 1 with form factor

\begin{tabular}{lllllll}
\hline & $e^{-} e^{+}$ & \multicolumn{5}{c}{$\mu^{-} \mu^{+}$} \\
& $b<15 \mathrm{fm}$ & $b>15 \mathrm{fm}$ & $b<15 \mathrm{fm}$ & $b>15 \mathrm{fm}$ & $b<15 \mathrm{fm}$ & $b>15 \mathrm{fm}$ \\
RHIC & $0.04 \%$ & $99.96 \%$ & $13.2 \%$ & $86.8 \%$ & $49.8 \%$ & $50.2 \%$ \\
LHC & $0.04 \%$ & $99.96 \%$ & $3.65 \%$ & $96.35 \%$ & $25 \%$ & $75 \%$ \\
& $b<20 \mathrm{fm}$ & $b>20 \mathrm{fm}$ & $b<20 \mathrm{fm}$ & $b>20 \mathrm{fm}$ & $b<20 \mathrm{fm}$ & $b>20 \mathrm{fm}$ \\
RHIC & $0.07 \%$ & $99.93 \%$ & $20.44 \%$ & $79.56 \%$ & $60.11 \%$ & $39.89 \%$ \\
LHC & $0.07 \%$ & $99.93 \%$ & $6.24 \%$ & $93.76 \%$ & $35.23 \%$ & $64.77 \%$ \\
& $b<25 \mathrm{fm}$ & $b>25 \mathrm{fm}$ & $b<25 \mathrm{fm}$ & $b>25 \mathrm{fm}$ & $b<25 \mathrm{fm}$ & $b>25 \mathrm{fm}$ \\
RHIC & $0.11 \%$ & $99.89 \%$ & $27.6 \%$ & $72.4 \%$ & $67.13 \%$ & $32.87 \%$ \\
LHC & $0.11 \%$ & $99.89 \%$ & $9.27 \%$ & $90.73 \%$ & $43.77 \%$ & $56.23 \%$ \\
& $b<30 \mathrm{fm}$ & $b>30 \mathrm{fm}$ & $b<30 \mathrm{fm}$ & $b>30 \mathrm{fm}$ & $b<30 \mathrm{fm}$ & $b>30 \mathrm{fm}$ \\
RHIC & $0.16 \%$ & $99.84 \%$ & $34.15 \%$ & $65.85 \%$ & $72.15 \%$ & $27.85 \%$ \\
LHC & $0.16 \%$ & $99.84 \%$ & $12.6 \%$ & $87.4 \%$ & $50.7 \%$ & $49.3 \%$ \\
\hline
\end{tabular}

at RHIC. On the other hand, when we change the radius $R$ from $6.1 \mathrm{fm}$ to $7.0 \mathrm{fm}$, the cross sections of the muon pairs decreases by about $5 \%$ at LHC and $11 \%$ at RHIC. A similar calculation shows that tauon production decreased by about $10 \%$ at LHC and $35 \%$ at RHIC. These calculations are tabulated in Table 3. From this calculation, we can conclude that the Wood-Saxon distribution function is more sensitive to the parameter $R$ compared to the parameter $a$. It also shows that the cross sections are very sensitive to the parameter $R$ at RHIC energies compared to the LHC energies. The proton distribution in the nucleus is well known. If the neutron surface is shifted outwards, for the proton surface this means that the skin depths of neutrons and protons are nearly equal $a_{n} \sim a_{p}$ and it is called bulk or skin type of neutron skin.
On the other hand, if the diffuseness of the neutron surface is greater than the proton, then $R_{n} \sim R_{p}$ and it is called surface or halo type of the neutron skin [32].

We have compared our results with the work in Ref. [33]. The two cross section results of the RHIC for $\mu^{-} \mu^{+}$production nearly agree; however, the $\tau^{-} \tau^{+}$cross section in our work approximately is 3 times larger than their results. Our LHC cross section results of $\mu^{-} \mu^{+}$and $\tau^{-} \tau^{+}$productions are slightly higher than their results. The authors in Ref. [33] used a Gaussian distribution form factor and the equivalent photon approximation to calculate the heavy-lepton pair production cross section.

When we compare our cross section results with Ref. [27], both calculations for $\mu^{-} \mu^{+}$production at RHIC ener- 


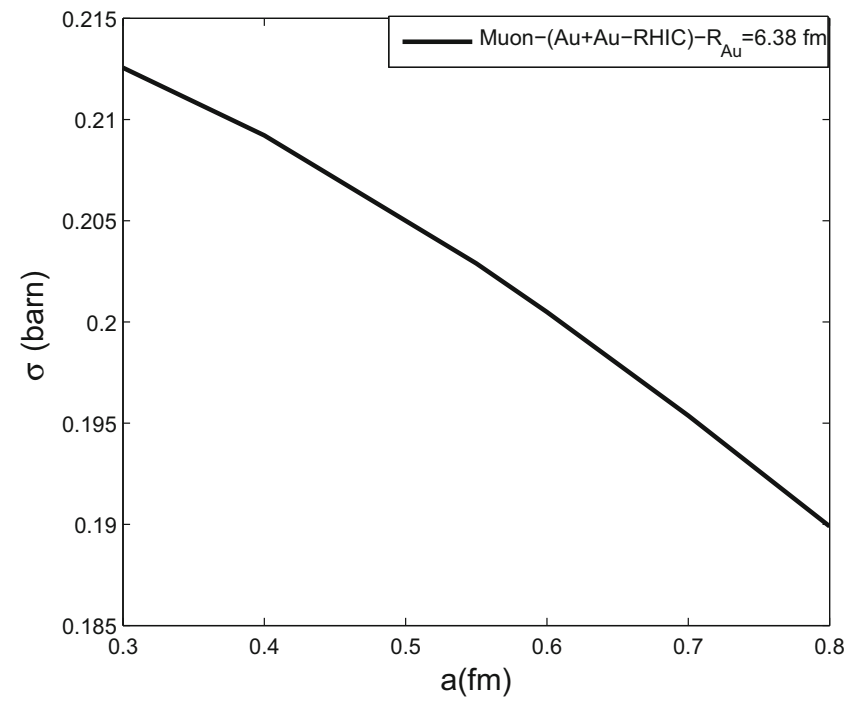

(a)

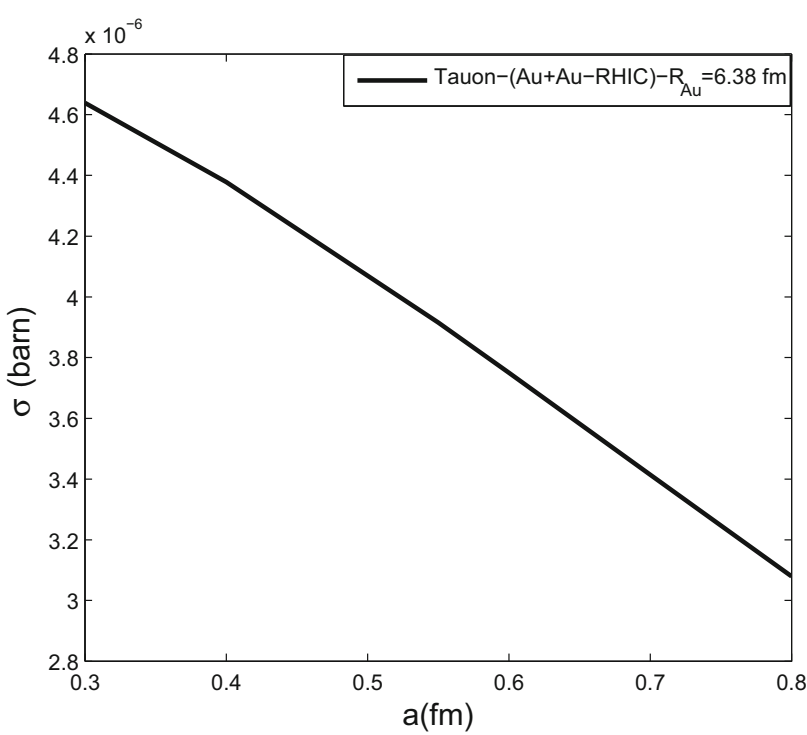

(c)

Fig. 10 The cross section of produced muon $\mathbf{a}$ as a function of the skin thickness $a$ at RHIC energy when $R=6.38 \mathrm{fm} \mathbf{b}$ as a function of $R$ at RHIC energy when the skin thickness $a=0.55 \mathrm{fm}$. The cross section

gies are almost similar with and without the form factor. At RHIC energies, the $\tau^{-} \tau^{+}$cross section with no form factor in our work approximately is 5 times lower than in Ref. [27], however, the results with the form factor are close to each other.

Our cross section results of $\mu^{-} \mu^{+}$rates at RHIC and LHC are about slightly lower than in Ref. [21], that is, using Born calculations at RHIC and LHC energies. Since the parameter $Z \alpha \approx 0.6$ is not small, Coulomb and unitary corrections

$\sigma_{\text {total }}=\sigma_{\text {two }- \text { photon }}+\sigma_{\text {Coulomb }}+\sigma_{\text {unitary }}$

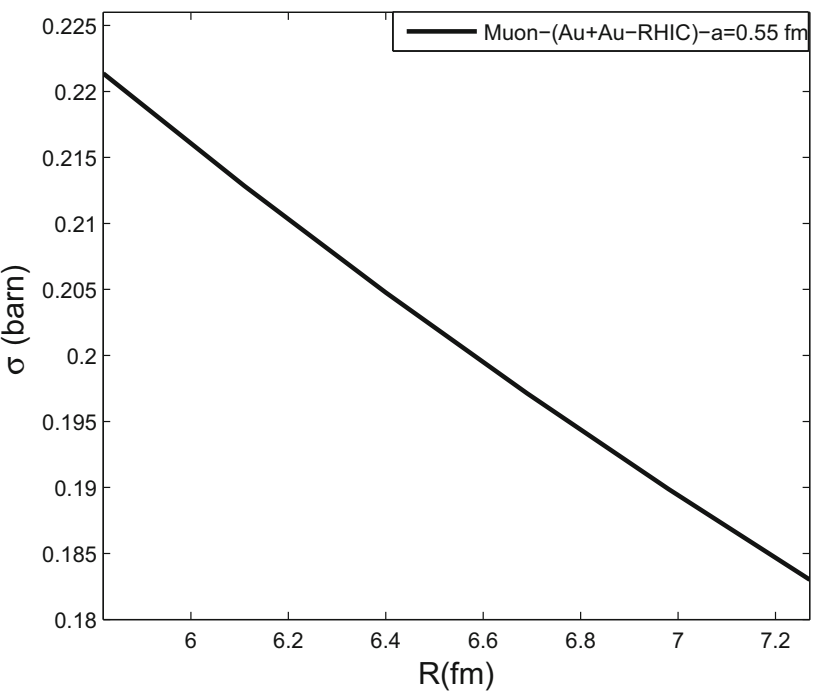

(b)

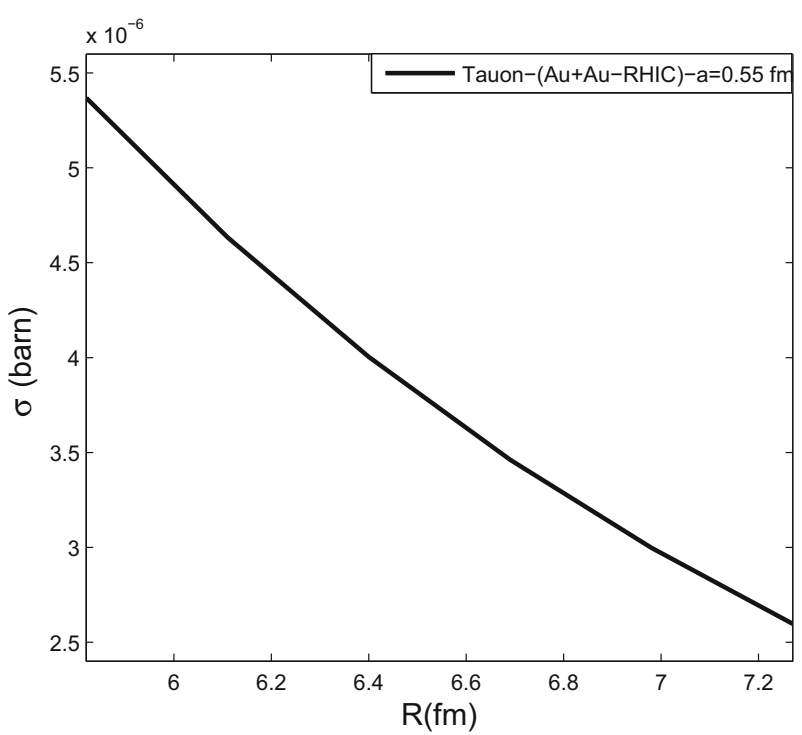

(d)

of the produced tauon $\mathbf{c}$ as a function of the skin thickness $a$ at RHIC energy when $R=6.38 \mathrm{fm} \mathrm{d}$ as a function of $R$ at RHIC energy when the skin thickness $a=0.55 \mathrm{fm}$

should be included in the calculations to obtain exact cross sections. The Coulomb correction corresponds to multiphoton exchange between the produced lepton pairs and nuclei [22], whereas the unitary correction corresponds to the exchange of light-by-light blocks between nuclei [11]. In Refs. $[11,21,22]$ the authors show that for electron-positron pair production, the Coulomb corrections to the Born cross section are large, whereas the unitary corrections are small.

On the other hand, for muon-pair production the Coulomb corrections to the Born cross section (corresponding to multiphoton exchange of the produced heavy-lepton pairs with 


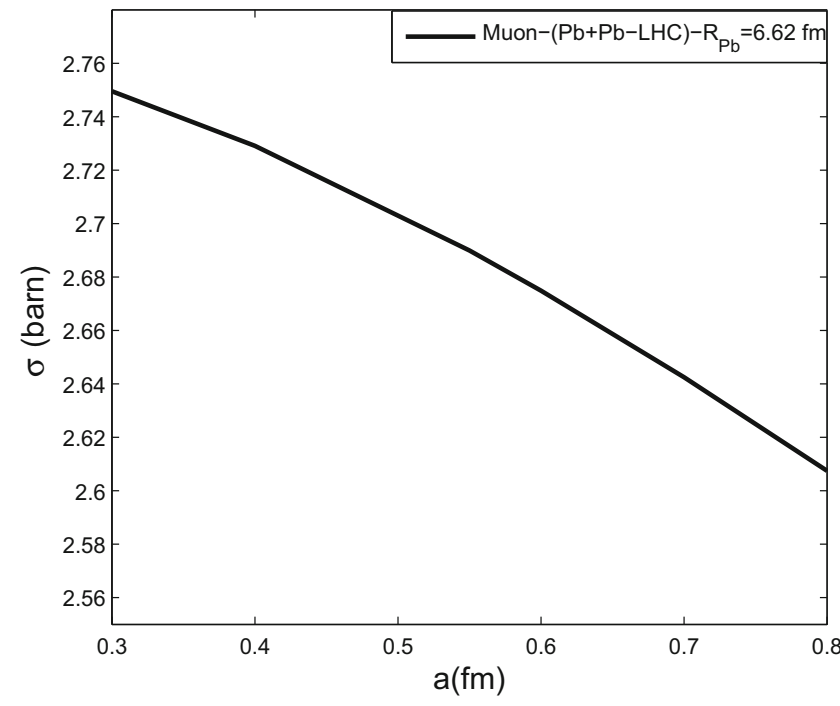

(a)

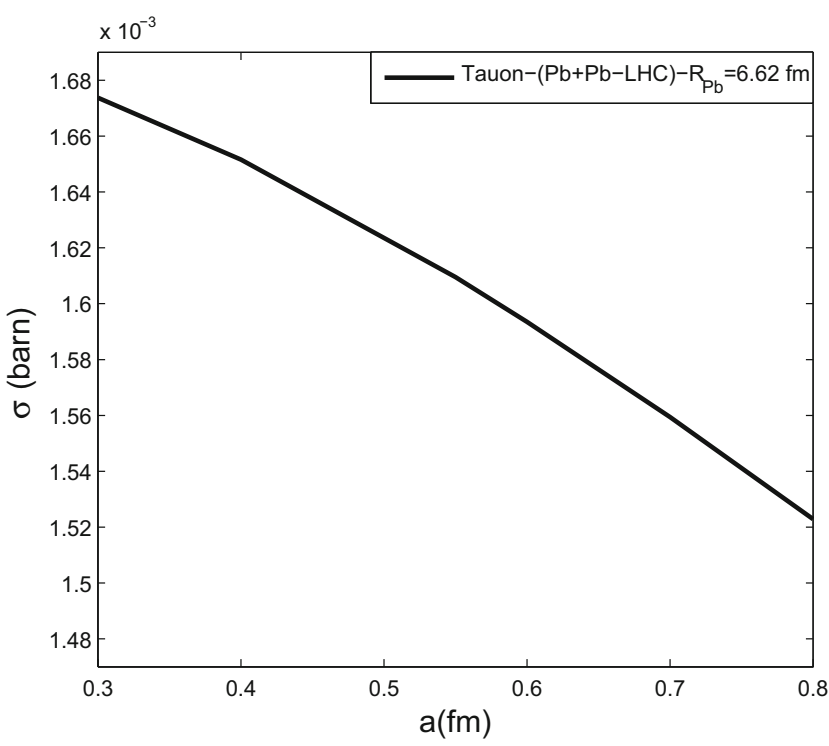

(c)

Fig. 11 The cross section of produced muon a as a function of skin thickness $a$ at LHC energy when $R=6.62 \mathrm{fm} \mathbf{b}$ as a function of $R$ at LHC energy when skin thickness $a=0.55 \mathrm{fm}$. The cross section of

Table 3 The ratio of the cross sections $(\Delta \sigma / \sigma)$ of the produced muon and tauon pairs. In the first column, the parameter $R$ is kept constant and the parameter $a$ is changed between $0.4 \mathrm{fm} \leq a \leq 0.6 \mathrm{fm}(\Delta a=$

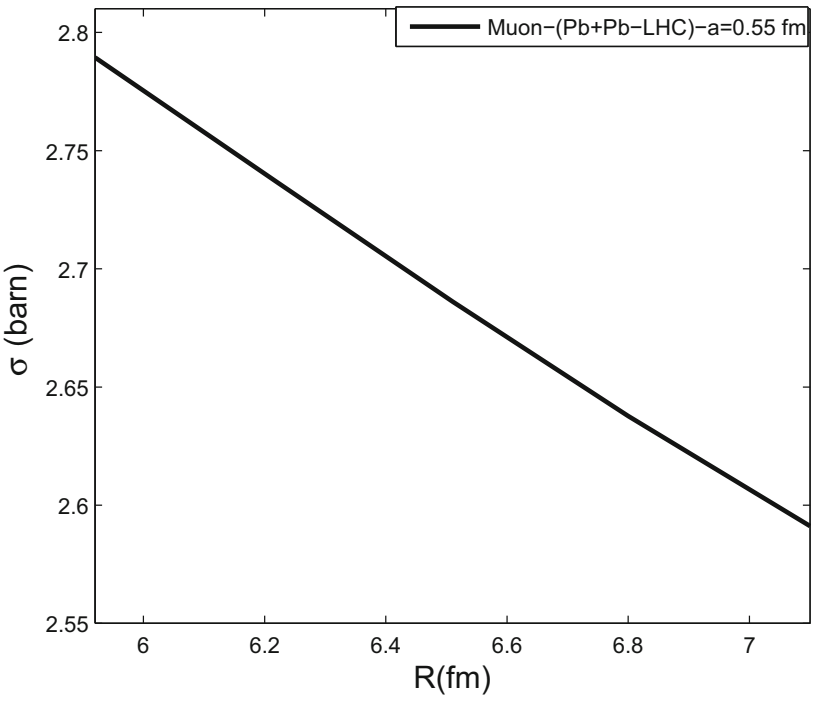

(b)

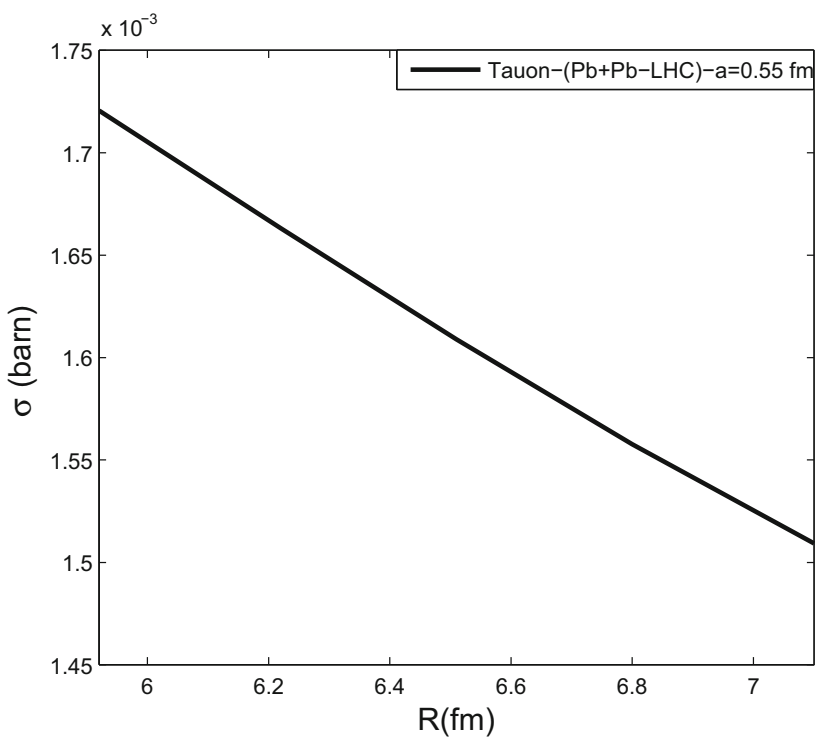

(d)

produced tauon $\mathbf{c}$ as a function of the skin thickness $a$ at LHC energy when $R=6.62 \mathrm{fm} \mathrm{d}$ as a function of $R$ at LHC energy when the skin thickness $a=0.55 \mathrm{fm}$

$0.2 \mathrm{fm}$ ). In the second column, the parameter $a=0.55 \mathrm{fm}$ is kept constant and the radius $R$ is changed between $6.1 \mathrm{fm} \leq R \leq 7.0 \mathrm{fm}$

\begin{tabular}{|c|c|c|c|}
\hline & & $\begin{array}{l}R_{A u}=6.38 f m, R_{P b}=6.62 f m \\
\Delta a=0.2 f m \\
\Delta \sigma / \sigma\end{array}$ & $\begin{array}{l}a=0.55 \mathrm{fm} \Delta R=0.9 \mathrm{fm} \\
\Delta \sigma / \sigma\end{array}$ \\
\hline \multirow[t]{2}{*}{$\mu^{-} \mu^{+}$} & RHIC & $4 \%$ & $11 \%$ \\
\hline & LHC & $2 \%$ & $5 \%$ \\
\hline \multirow[t]{2}{*}{$\tau^{-} \tau^{+}$} & RHIC & $14 \%$ & $35 \%$ \\
\hline & LHC & $4 \%$ & $10 \%$ \\
\hline
\end{tabular}


the nuclei) are small, since the form factor eliminates all the exchange photon momenta for the range of $k_{\perp}^{2}>1 / R^{2}$. Therefore the cross section is suppressed by $(Z \alpha)^{2} /(R M)^{2}$ where $M$ is the mass of the heavy lepton and $R$ is the radius of the nuclei. The calculation shows that the contribution of the Coulomb correction is less than $1 \%$ of the total cross sections [21].

For the unitary corrections (corresponding to the exchange of light-by-light blocks between nuclei), we can consider both the exclusive (the heavy-lepton pair production without any electron-positron pair production) and the inclusive (the heavy-lepton pair production with several electron-positron pair production) heavy-lepton pair production. The authors find that for exclusive muon-pair production for $\mathrm{Pb}+\mathrm{Pb}$ collisions at LHC there are about $-50 \%$ unitary corrections to the Born cross sections; however, for inclusive muon-pair production, the unitary correction is very small. In this work, we have repeated the calculations and our results for the heavylepton pair productions coincide with the above work.

All these calculations are done for all ranges of momenta and energies of the heavy leptons. However, for a given detector, electron and muon pairs can be measured with some restricted momenta. For example, the ALICE detector can measure only forward muons with pseudorapidity $4<y<5$ and $p_{t}>2 \mathrm{GeV}$. Similarly, the CMS detector in LHC and the STAR and PHENIX detectors in RHIC have some rapidity cuts and restricted transverse momenta. In Ref. [3] the authors made some predictions for electromagnetic exclusive muon-pair productions for the above detectors with the relevant restrictions.

The exclusive production of mesons, pairs of quark and antiquark particles, pairs of leptons in ultraperipheral heavyion collisions (UPC) have been investigated by several authors [34-36]. Some of the authors calculated the neutron emission from the excited nuclear system within the HauserFeshbach formalism and compared the results with the recent experimental data of PHENIX, PHOBOS, BRAHMS, and ALICE collaborations. Those methods can also be applied to the nuclear excitations with lepton-pair production. In our future work, we are planning to do calculations about nuclear excitations with mainly giant dipole resonance with heavylepton pair productions with the kinematic restrictions of the above detectors and compare with the experimental results.

\section{Conclusions}

In this work, we investigate the contribution of the WoodSaxon nuclear form factors on the heavy-lepton pair production. We study the pair productions for the RHIC and LHC energies for the $\mathrm{Au}$ and $\mathrm{Pb}$ nucleus. Even though electronpair production is not strongly affected by the form factors, the calculations show that nuclear form factors play important role for the muon- and tauon-pair production. At RHIC and LHC energies, muon-pair production is reduced by factors of about 3 and 2 by the $\mathrm{Au}$ and $\mathrm{Pb}$ form factors, respectively. On the other hand, at RHIC and LHC energies, tauon pair production is reduced by factors of about 100 and 5 by the $\mathrm{Au}$ and $\mathrm{Pb}$ form factors, respectively. These calculations show that tauon-pair production is very sensitive to the form factors at the RHIC energies. We can conclude that muon-pair production in peripheral heavy-ion collisions is coherent on distances greater than the dimensions of the nucleus. However, although for the LHC energies the tauon-pair production is coherent over distances of the dimension of the nucleus, for the RHIC energies this coherence breaks down. Since the mass of the tauon is very large when compared to the muon, incoherent pair production of tauon is dominant at the RHIC energies. When the energy is increased, the coherence pair production is restored. We expect that this result is also valid for the other heavy particles.

We have also investigated the effect of the neutron distributions in the nucleus to the cross section of the heavy-lepton pair production. The neutron skin can be produced in two ways: The first type is as a result of the shift of the neutron surface in relation to the proton one (the bulk or "skin" type $a_{n} \sim a_{p}$ ), and the second type is due to the differences in the surface thickness of proton and neutron matter in a nucleus (the surface or "halo" type $R_{n} \sim R_{p}$ ). In general, both types of mechanisms contribute to the creation of the neutron skin. We have changed the parameters $a$ and $R$ in the Wood-Saxon form factor, and our calculations show that the Wood-Saxon distribution function is more sensitive to the parameter $R$ compared to the parameter $a$.

Open Access This article is distributed under the terms of the Creative Commons Attribution 4.0 International License (http://creativecomm ons.org/licenses/by/4.0/), which permits unrestricted use, distribution, and reproduction in any medium, provided you give appropriate credit to the original author(s) and the source, provide a link to the Creative Commons license, and indicate if changes were made.

Funded by SCOAP . $^{3}$

\section{References}

1. A. Belkacem, A.H. Sorensen, Phys. Rev. A 57, 3646 (1998)

2. U.D. Jentschura, V.G. Serbo, Eur. Phys. J. C 64, 309 (2009)

3. M. Klusek-Gawenda, A. Szczurek, Phys. Rev. C 82, 014904 (2010)

4. A.J. Baltz, Phys. Rev. C 80, 034901 (2009)

5. A.J. Baltz, Y. Gorbunov, S.R. Klein, J. Nystrand, Phys. Rev. C 80, 044902 (2009)

6. I. Angeli, At. Data Nucl. Data Tabl. 87, 185 (2004)

7. S. Abrahamyan et al., Phys. Rev. Lett. 108, 112502 (2012)

8. G. Racah, Nuovo Cimento 14, 93 (1937)

9. C.F. von Weizsacker, Z. Phys. 88, 612(1934). (E. J. Williams, Phys. Rev. 45, 730 (1934))

10. C. Bottcher, M.R. Strayer, Phys. Rev. D 39, 1330 (1989) 
11. M.C. Güçlü, J. Li, A.S. Umar, D.J. Ernst, M.R. Strayer, Ann. Phys. 272, 7 (1999)

12. K. Hencken, D. Trautmann, G. Baur, Phys. Rev. C 59, 841 (1999)

13. C. Best, W. Greiner, G. Soff, Phys. Rev. A 46, 261 (1992)

14. J. Eichler, Phys. Rev. Lett. 75, 3653 (1995)

15. A.J. Baltz, M.J. Rhoades-Brown, J. Weneser, Phys. Rev. A 48, 2002 (1993)

16. A.J. Baltz, M.J. Rhoades-Brown, J. Weneser, Phys. Rev. Lett. 78, 1231 (1997)

17. G. Baur, K. Hencken, D. Trautmann, Phys. Rep. 453, 1 (2007)

18. M.C. Güçlü, J.C. Wells, A.S. Umar, M.R. Strayer, D.J. Ernst, Phys. Rev. A 51, 1836 (1995)

19. M.Y. Şengül, M.C. Güçlü, S. Fritzsche, Phys. Rev. A 80, 042711 (2009)

20. M.Y. Şengül, M.C. Güçlü, Prog. Part. Nucl. Phys. 67, 612 (2012)

21. K. Hencken, E.A. Kuraev, V.G. Serbo, Phys. Rev. C 75, 034903 (2007)

22. DYu. Ivanov, A. Schiller, V.G. Serbo, Phys. Lett. B 454, 155 (1999)

23. R.N. Lee, A.I. Milstein, V.G. Serbo, Phys. Rev. A 65, 022102-1 (2002)

24. C.A. Bertulani, G. Baur, Phys. Rep. 163, 299 (1988)

25. V.B. Berestetskii, E.M. Lifshitz, L.P. Pitaevskii, Relativistic Quantum Field Theory (Pergamon Press, New York, 1979)
26. J. Eichler, W.E. Meyerhof, Relativistic Atomic Collisions (Academic Press, California, 1995)

27. C. Bottcher, M.R. Strayer, J. Phys. G Nucl. Part. Phys. 16, 975 (1990)

28. R.C. Barrett, D.F. Jackson, Nuclear Sizes and Structure (Oxford University Press, New York, 1977)

29. S.R. Klein, J. Nystrand, Phys. Rev. C 60, 014903 (1999)

30. W. Magnus, F. Oberhettinger, Special Functions of Mathematical Physics (Chelsea Publ. Co, NY, 1954)

31. M.Y. Şengül, Int. J. Mod. Phys. E 25, 1650006 (2016)

32. M. Centelles, X. Roca-Maza, X. Vinas, M. Warda, Phys. Rev. C 82, 054314 (2010)

33. M. Vidovic, M. Greiner, G. Soff, J. Phys. G Nucl. Part. Phys. 21, 545 (1995)

34. G. Baur, K. Hencken, D. Trautmann, S. Sadovsky, Y. Kharlov, Phys. Rep. 364, 359 (2002)

35. C.A. Bertulani, S.R. Klein, J. Nystrand, Ann. Rev. Nucl. Part. Sci. 55, 271 (2005)

36. M. Klusek-Gawenda, M. Ciemala, W. Schafer, A. Szczurek, Phys. Rev. C 89, 054907 (2014) 